\title{
Surface Scattering Near Grazing Angles: The Distorted Wave Born Approximation for Rough Surfaces
}

G Ljungdahl and S W Lovesey

June 1995 
(c) Council for the Central Laboratory of the Research Councils 1995

Enquiries about copyright, reproduction and requests for

additional copies of this report should be addressed to:

The Central Laboratory for the Research Councils

Library and Information Services

Rutherford Appleton Laboratory

Chilton

Didcot

Oxfordshire

OX11 OQX

Tel: 01235445384 Fax: 01235446403

E-mail library@rl.ac.uk

ISSN 1358-6254

Neither the Council nor the Laboratory accept any responsibility for loss or damage arising from the use of information contained in any of their reports or in any communication about their tests or investigations. 


\title{
Surface Scattering Near Grazing Angles: The Distorted Wave Born Approximation for Rough Surfaces
}

Gösta Ljungdahl and Stephen W. Lovesey, ISIS Facility, Rutherford Appleton Laboratory, Oxon OX11 0QX, U.K.

PACS number: 6820

\begin{abstract}
The scattering of radiation by mildly rough surfaces of non-absorbing materials is analyzed in the distorted wave Born approximation wherein the roughness is treated as a perturbation. The basic formulae for the scattering cross-section are derived and corresponding expressions for the reflectivity and transmissivity are given. It is shown that off-specular scattering is proportional to the Fourier transform of an autocorrelation function involving phase factors produced when the actual surface deviates from a flat reference surface. In the specular direction the Fourier transform reduces to a simple surface average. The theory is then applied to periodic surfaces where the Fourier transform is reduced to a weighted sum of delta-functions. Numerical reflectivities are given for a piecewise rectangular and a sinusoidal grating. For such surfaces it is shown that plausible results are obtained even when the deviations are large. Random roughness is considered and explicit results are given for a surface with a Gaussian distribution of the height variation. It is demonstrated that the surface roughness for this type of surface must be small for our perturbative technique to be valid. For off-specular scattering numerical reflectivities are given in the limit of small deviations or small wave vector transfers where the scattering is proportional to the Fourier transform of the autocorrelation function of the deviations themselves.
\end{abstract}




\section{Introduction}

Beams of particles are used in scattering experiments to study spatial structure on a scale of detail that ranges from sub-atomic particles to aggregates made of atoms and molecules. From the interpretation of these experiments there is empirical evidence that a class of phenomena, which arise from multiple-scattering events, can legitimately be described in terms of concepts familiar from our understanding of light scattering. These concepts are usually embraced by the title (Euclidean) geometrical optics, and they include a refractive index and reflection and refraction. Our interest lies with scattering of radiation by surfaces and interfaces at very shallow incident angles, near the grazing angle condition at which total external reflection occurs.

By and large, the scattered signal contains just a modest amount of information about the surface layers of the target. So, usually the recorded signal is quite properly interpreted in terms of the bulk properties of the target. However, sensitivity to the surface layers is greatly enhanced if scattering takes place under conditions close to total external reflection. At a grazing angle small enough to satisfy this condition, the surface layer is fully illuminated by the incident radiation. One finds for these conditions that the diffraction pattern does indeed reveal the structure of the surface region in preference to the bulk interior. Here, we address the question of how to interpret scattering at grazing angles from a surface that is not an optical flat, i.e. a rough surface. The experiments which motivate our work aim to provide information at an atomic level of detail on air-liquid or solid interfaces, etc.

The chosen method of investigation was proposed by Vineyard [1]. It hinges on expressing as a perturbation in scattering theory the deviation of the real, rough surface from an optical flat. The systematic expansion in powers of the perturbation of the scattering amplitude is in our work terminated at the first level. In the theoretical development the basic building blocks are the wave functions for reflection and refraction by an optical flat. These functions are the appropriate Fresnel wave functions. Of course, in the context of scattering theory this amounts to finding the solution to a multiple scattering problem via the dynamical theory of diffraction. Regrettably, it is nigh impossible to extend calculations using the dynamical theory to 
rough surfaces. A way round the impasse is the method proposed by Vineyard [1], in which one starts from an exact solution for scattering by two potential fields. Ultimately, one potential represents an optical flat and the second is the deviation of the optical flat from the real surface of physical interest. A review of theoretical work that stems from Vineyard's method is given by Dosch [2] together with experimental results obtained using $\mathrm{x}$-ray and neutron beams.

The present formulation, for the case of neutrons scattered by a mildly rough surface, yields formulae for the specular and off-specular (diffuse) reflectivities that are considerably simpler in their algebraic structures than previously reported formulae $[3,4]$. Our formulae are evaluated for a few examples, chosen to illustrate features of roughness induced effects observed near the grazing angle of incidence.

We start our report, in $\$ 2$, with a derivation of the reflectivity for an optical flat. This exercise serves as a valuable orientation to the modifications induced by surface roughness which are taken up in $\S 3$. The work is based, following Vineyard [1], on the conventional distorted wave formulation of scattering theory; the latter for completeness is outlined in an appendix. The modification to the scattering amplitude is calculated with this formulation to first order in the deviation of the rough surface from an optical flat. This level of approximation should suffice for a mildly rough surface. Since the work for the rough surface makes use of the Fresnel solutions for an optical flat the required results are gathered in Appendix A. Our findings from scattering by two model surfaces, and random surface structures are given in $\S \S 4,5$, respectively. Section 6 contains a discussion and the main conclusions.

\section{Reflectivity of an Optical Flat}

By way of an orientation to the methodology used later to discuss scattering by a rough surface we use it first to calculate the reflectivity of an optical flat. The conventional derivation of the reflectivity, $R$, for this surface is reviewed by Sears [5], and the result is listed in Appendix A of this paper. 
Our starting point is scattering theory, which is précised in Appendix B. The key quantity is the matrix element of the t-matrix given by (B.4). For an optical flat the potential field in (B.4) is,

$$
\begin{aligned}
V & =V_{0}, z \leq 0 \\
& =0, z>0,
\end{aligned}
$$

where $V_{0}$ is the material optical potential and the coordinates are defined in fig. (1). A convincing derivation of $V_{\mathrm{o}}$ is hard to find [5]. The core problem is relatively easy to state, scilicet, to progress logically from quantum mechanics at the atomic-level of detail to a scale of bulk thermodynamic quantities, e.g. the material optical potential and the corresponding coherent wave function. At a technical level, the challenge is to treat adequately the multiple scattering intrinsic in optical phenomena. The result,

$$
V_{\mathrm{o}}=\frac{(\hbar k)^{2}}{2 m}\left(1-n^{2}\right)
$$

where $\left(\hbar^{2} / 2 m\right)=2.08 \mathrm{meV} \AA^{2}$ for neutrons, can be regarded as a tenet of optics. In this instance, the refractive index, $n$, is usually less than unity. The magnitude of the incident wave vector, $k$, is related to the components of the incident and refracted wave vectors normal to the plane, $-k_{z}$ and $-k_{z}^{t}$, respectively, through (see fig. (1) and Appendix A),

$$
k^{2}\left(1-n^{2}\right)=k_{z}^{2}-\left(k_{z}^{t}\right)^{2}
$$

Since $V_{0}$ does not depend on the position variable, $\mathbf{r}$, it factors out of the t-matrix (B.4), for which we get,

$$
\left\langle\chi_{2}^{(-)}|V| \varphi_{1}\right\rangle=V_{0} \int_{z \leq 0} \mathrm{~d} \mathbf{r}\left\{\chi_{2}^{(-)}(\mathbf{r})\right\}^{*} \varphi_{1}(\mathbf{r})
$$


In this expression, $\chi_{2}^{(-)}(\mathbf{r})$ is a particular Fresnel wave function with the explicit form,

$$
\chi_{2}^{(-)}(\mathbf{r})=t *\left(-\mathbf{k}_{2}\right) \exp \left(\mathbf{i r} \cdot \mathbf{k}_{2}^{t}\right) ; \quad z \leq 0
$$

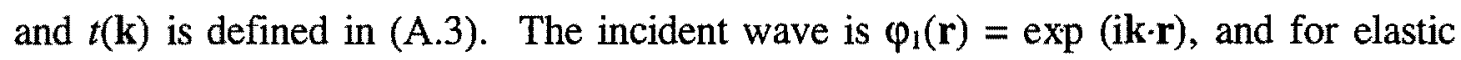
scattering the magnitudes of $\mathbf{k}$ and $\mathbf{k}_{2}$ are equal. Using the definitions, $\mathbf{k}=\left(k_{x}, k_{y},-k_{z}\right), \mathbf{k}_{2}^{t}=\left(k_{2 x}, k_{2 y}, k_{2 z}^{t}\right)$ and the notation $\mathrm{K}=\left(k_{2 x}-k_{x}, k_{2 y}-k_{y}\right)$, the matrix element is,

$$
\left\langle\chi_{2}^{(-)}|V| \varphi_{1}\right\rangle=\left\{\mathrm{i} V_{0} t\left(-\mathbf{k}_{2}\right) /\left(k_{z}+k_{2 z}^{t}\right)\right\} \int \mathrm{d} \rho \exp (-\mathrm{ik} \cdot \rho)
$$

Here, the integration with respect to the two-dimensional vector, $\mathrm{p}=(x, y)$, extends over the area, $A_{0}$, illuminated by the radiation. Also on the subject of integration limits, in arriving at (2.4) the z-integration has been extended to infinity. The licence for this step lies in the certain knowledge that in an application the transmitted wave will decay through absorption. The assumption is that, the characteristic decay length is small compared to the thickness of the sample, so the sample thickness is not a relevant parameter in the problem.

The corresponding elastic cross-section follows from (B.5), namely,

$$
(\mathrm{d} \sigma / \mathrm{d} \Omega)=\left(m / 2 \pi \hbar^{2}\right)^{2}\left|\left\langle\chi_{2}^{(-)}|V| \varphi_{1}\right\rangle\right|^{2}
$$

Combining (2.2), (2.3) and (2.4) in (2.5) leads directly to, 


$$
(\mathrm{d} \sigma / \mathrm{d} \Omega)=(1 / 2 \pi)^{2}\left|\frac{k_{2 z}\left[k_{z}^{2}-\left(k_{z}^{t}\right)^{2}\right]}{\mid\left(k_{z}+k_{2 z}^{t}\right)\left(k_{2 z}+k_{2 z}^{t}\right)}\right|^{2} \quad\left|\int \mathrm{d} \rho \exp (-\mathrm{i} \kappa \cdot \rho)\right|^{2} .
$$

In arriving at this result we have made use of (A.3) for $t(\mathbf{k})$ together with $t(\mathbf{k})=t(-\mathbf{k})$.

It remains to estimate the two-dimensional integral in (2.6). In the limit of a very large illuminated area the integral is very small except in the special case $\kappa=0$ where it has a value proportional to the size of the surface, i.e. the integral yields Bragg's law for diffraction. In the extreme limit,

$$
\left|\int \mathrm{d} \rho \exp (-\mathrm{i} \kappa \cdot \rho)\right|^{2}=(2 \pi)^{2} A_{\mathrm{o}} \delta(\kappa)
$$

where $\delta(\kappa)=\delta\left(\kappa_{x}\right) \delta\left(\kappa_{y}\right)$ is a two-dimensional Dirac delta-function which vanishes if its argument is not zero. One way to derive (2.7) is to note first that, for an infinite area, the integral is a standard representation of the delta-function, $\delta(\kappa)$. Hence, its square is proportional to $\delta(\kappa)$, as shown, and the coefficient of proportionality is obtained by integrating both sides with respect to $\kappa$. Further discussion of this integral is found in $\$ 3.2$.

The conditions $\mathrm{\kappa}=0$, from the action of the delta function, and $k=k_{2}$ (elastic scattering) lead to the result $k_{z}=k_{2 z}$, together with $k_{z}^{t}=k_{2 z}^{t}$. Employing these results and (2.7) leads to the cross-section,

$$
(\mathrm{d} \sigma / \mathrm{d} \Omega)=A_{0} \delta(\kappa)\left|k_{z} r(\mathbf{k})\right|^{2},
$$

where $r(\mathbf{k})$ is a Fresnel coefficient, defined in (A.3). The final step in the calculation of the reflectivity is the relation,

$$
R=\left(1 / A_{0} \sin \theta\right) \int \mathrm{d} \Omega(\mathrm{d} \sigma / \mathrm{d} \Omega),
$$


where $\theta$ is the angle of incidence defined in fig. (1). For the case of elastic scattering, the element of solid angle,

$$
\mathrm{d} \Omega=\left(\mathrm{d} k_{2 x} \mathrm{~d} k_{2 y}\right) / k^{2} \sin \theta=\left(\mathrm{d}_{x} \mathrm{~d} \kappa_{y}\right) / k^{2} \sin \theta
$$

On combining this result in (2.9) with (2.8), and $k_{z}=k \sin \theta$ yields,

$$
R=|r(\mathbf{k})|^{2}
$$

which is the result quoted in Appendix A. The corresponding transmissivity, $T$, is obtained from the conservation of flux, namely,

$$
T=(1-R)
$$

\section{Scattering by a Rough Surface}

We define the material surface by a real function $\zeta(x, y)$ that lies in the half-space $z \leq 0$, as shown in fig. (2). The plane through $z=0$ defines an optical flat, which is the subject of the previous section.

Scattering by the rough material surface is described by the application of the distorted wave formulation of scattering theory. (The Rayleigh criterion of roughness is provided in Appendix A.) We will use the first level of approximation in which the scattering amplitude is linear in the potential field generated by the deviation of the rough surface from an optical flat. In the notation used in Appendix B, this potential field is denoted by $V_{\mathrm{b}}$, and $V_{\mathrm{a}}$, the potential field (2.1) for an optical flat, is known as the distorting potential field which is responsible for the Fresnel wave functions. To be specific, 


$$
\begin{aligned}
V_{\mathrm{b}} & =-V_{\mathrm{o}} ;-\zeta(x, y) \leq z \leq 0 \\
& =0, \text { elsewhere. }
\end{aligned}
$$

Henceforth, we adopt a convention, that will hopefully not cause confusion, in which the argument of the function $\zeta(x, y)$ is not explicitly shown.

The $t$ - matrix for the surface roughness is given by (B.11), in which the righthand side is the working formula at our chosen level of approximation. From (3.1) and (B.11),

$$
\left\langle\chi_{2}^{(-)}\left|V_{\mathrm{b}}\right| \chi_{1}^{(+)}\right\rangle=-V_{\mathrm{o}} \int \mathrm{d} \rho \int_{-\zeta}^{0} \mathrm{~d} z\left\{\chi_{2}^{(-)}(\mathbf{r})\right\}^{*} \chi_{1}^{(+)}(\mathbf{r})
$$

In this expression, we have imported some of the notation from $\$ 2$ where we have already used the Fresnel wave function $\chi_{2}^{(-)}(\mathbf{r})$. The second Fresnel wave function in (3.2) is,

$$
\chi_{1}^{(+)}(\mathbf{r})=t(\mathbf{k}) \exp \left(\mathbf{i r} \cdot \mathbf{k}^{t}\right) ; \quad z \leq 0
$$

with $\mathbf{k}^{t}=\left(k_{x}, k_{y},-k_{z}^{t}\right)$. (Note that we continue to use the simplification adopted in $\$ 2$ of omitting a subscript " 1 " on the wave vector $\mathbf{k}^{\natural}$.) Simple algebra leads to the result,

$$
\left\langle\chi_{2}^{(-)}\left|V_{\mathrm{b}}\right| \chi_{1}^{(+)}\right\rangle=t\left(-\mathbf{k}_{2}\right) t(\mathbf{k})\left(V_{\mathrm{o}} / \mathrm{i} q_{\mathrm{l}}\right) \int \mathrm{d} \rho\left\{1-\exp \left(\mathrm{i} q_{\mathrm{l}} \zeta\right)\right\} \exp (-\mathrm{i} \mathbf{k} \cdot \rho)
$$

where $q_{t}=\left(k_{z}^{t}+k_{2 z}^{t}\right)$, and $\zeta=\zeta(x, y)$. It is prudent to develop (3.3) separately for the two cases of specular and off-specular scattering. 


\subsection{Specular Scattering}

The so-called specular condition is contained in the relations $\mathrm{\kappa}=0, k_{z}=k_{2 z}$, and $k_{z}^{t}=k_{2 z}^{t}$. Referring to (3.3) the specular condition means $q_{t}=2 k_{z}^{t}$. With a view to further workings, the t-matrix is conveniently written as,

$$
\left\langle\chi_{2}^{(-)}\left|V_{\mathrm{b}}\right| \chi_{1}^{(+)}\right\rangle=\left(V_{\mathrm{o}} / \mathrm{i} q_{t}\right) t^{2}(\mathbf{k}) A_{\mathrm{o}}\left\{1-\left\langle\exp \left(\mathrm{i} q_{t} \zeta\right)\right\rangle\right\}
$$

where $<\ldots>$ denotes a simple average over the illuminated surface, of area $A_{0}$, which is defined by,

$$
\left\langle\exp \left(\mathrm{i} q_{\imath} \zeta\right)\right\rangle=\left(1 / A_{\mathrm{o}}\right) \int \mathrm{d} \rho \exp \left(\mathrm{i} q_{\imath} \zeta\right)=1-\langle S\rangle
$$

where the second equality defines a function $S$. For specular scattering, $\langle S\rangle$ is an explicit function of $k_{z}$.

Evaluated in the distorted wave Born approximation, the t-matrix for specular scattering is the sum of (2.4) and (3.4). After a little rearrangement the result can be reduced to,

$$
\begin{aligned}
&\left\langle\varphi_{2}|V| \psi_{1}^{(+)}\right\rangle \approx\left\langle\chi_{2}^{(-)}\left|V_{\mathrm{a}}\right| \varphi_{1}\right\rangle+\left\langle\chi_{2}^{(-)}\left|V_{\mathrm{b}}\right| \chi_{1}^{(+)}\right\rangle \\
&=\left(\mathrm{i} A_{\mathrm{o}} \hbar^{2} k_{z} r(\mathbf{k}) / m\right)\left\{1-\left(k_{z} / k_{z}^{t}\right)\langle S\rangle\right\} .
\end{aligned}
$$

Here, the Fresnel coefficient, $r(\mathbf{k})$, is defined in (A.3) and $k_{z}^{t}$ is determined by (A.1) from a knowledge of $k_{z}$, the grazing angle and refractive index. If the latter is less than unity, $k_{z}^{t}$ is purely imaginary at a sufficiently small grazing angle. In (3.6), the quantity in curly brackets can be viewed as a renormalization factor, applied to the bare Fresnel 
coefficient, which is created by the departure of the material surface from an optical flat.

Looking back to the derivation in $\$ 2$ of the reflectivity of an optical flat it follows that, from the result (3.6) the reflectivity for a rough surface, calculated within the first Born approximation, is,

$$
R_{s}=\left|r(\mathbf{k})\left\{1-\left(k_{z} / k_{z}^{\prime}\right)\langle S\rangle\right\}\right|^{2}
$$

A subscript " $\mathrm{s}$ " has been added to the symbol $R$ to denote the fact that it is the reflectivity for specular scattering. Later, we will introduce the reflectivity for offspecular (diffuse) scattering, $R_{\mathrm{d}}$. Of course, $R_{\mathrm{s}}, R_{\mathrm{d}}$ and the corresponding transmissivities $T_{\mathrm{s}}, T_{\mathrm{d}}$ satisfy the relation,

$$
R_{\mathrm{s}}+T_{\mathrm{s}}+R_{\mathrm{d}}+T_{\mathrm{d}}=1
$$

and the magnitude of each of the four positive quantities has an upper bound of unity.

A useful insight to the correction in (3.7) induced by the roughness can be found by taking the limit to the critical angle of incidence, $\theta_{c}$. In this instance, $k_{z}^{t} \rightarrow 0$ and (3.7) achieves the value,

$$
R_{\mathrm{s}}=\left|\left(1+2 \mathrm{i} k_{z}\langle\zeta\rangle\right)\right|^{2} ; \quad \theta \rightarrow \theta_{\mathrm{c}}^{+}
$$

In general, $\langle\zeta\rangle$ is both purely real and finite, and it is a measure of the distance of the rough surface below the reference optical flat, cf. fig. (2). The interpretation given to the roughness induced correction in (3.9) is that it is the first term in the expansion of a phase factor, $\exp \left(2 \mathrm{i} k_{z}\langle\zeta\rangle\right)$. The remaining terms in the expansion of the phase factor in powers of $\langle\zeta\rangle$ are generated by the higher-order terms in the t-matrix beyond the Born approximation on which (3.9) is based. On accepting this interpretation, we are lead to the satisfactory result $R_{\mathrm{s}} \rightarrow 1$ for $\theta \rightarrow \theta_{\mathrm{c}}^{+}$. 
For the specular transmissivity in (3.8) we follow a similar procedure using (A.9) rather than (A.7). We find,

$$
T_{\mathrm{s}}=\left|t(\mathbf{k})+\left\{\left(\frac{1-t(\mathbf{k})}{2 k_{z}^{t}}\right)\langle S\rangle-\mathrm{i}\langle\zeta\rangle\right\} k_{z}\left(k_{z}^{t}-k_{z}\right) / k_{z}^{t}\right|^{2} .
$$

It is interesting to note that, $T_{\mathrm{s}}$ contains a term which is linear in the mean value of the coordinate $\zeta$ which defines the surface.

\subsection{Off-Specular Scattering}

As a first task we address the appropriate interpretation of the Fraunhofer diffraction in the amplitude (3.3) that is represented by the integral,

$$
\int d \rho \exp (-\mathrm{i} \kappa \cdot \rho)
$$

where the integration spans the illuminated area. (We refer to the scattering as Fraunhofer diffraction because the distance from the sample to the point of observation is large compared with the dimension of the illuminated area.) If the product of $K$ and the dimension of the illuminated area is much greater than unity the integral in question is negligible for all $\kappa$ except $\kappa=0$, and in the extreme limit, where the product tends to infinity, one recovers a strict selection rule,

$$
\underset{\kappa^{2} A_{o} \rightarrow \infty}{\lim } \int d \rho \exp (-\mathrm{i} \kappa \cdot \rho)=(2 \pi)^{2} \delta(\kappa)
$$

The last result has been encountered in (2.7). For a finite area, $A_{o}=f h$, one readily finds in place of (3.11), 


$$
\begin{gathered}
\int d \rho \exp (-\mathrm{i} \kappa \cdot \rho)=\left(4 / \mathrm{\kappa}_{x} \kappa_{y}\right) \sin \left(\frac{1}{2} f \kappa_{x}\right) \sin \left(\frac{1}{2} h \kappa_{y}\right) \\
=A_{0} j_{0}\left(\frac{1}{2} f \kappa_{x}\right) j_{0}\left(\frac{1}{2} h \kappa_{y}\right) .
\end{gathered}
$$

The standard representation of the Dirac delta-function,

$$
\lim _{\xi \rightarrow \infty}\{\sin (x \xi) / x \pi\}=\delta(x)
$$

enables us in the appropriate limit to obtain from (3.12) the result (3.11). Furthermore, (3.12) and (3.13) can be used to construct another argument that supports the result quoted in (2.7). Returning to (3.11), in the event that $\zeta(x, y)$ contains a monochromatic component a selection rule will apply to its Fourier component, e.g. the result (4.2).

In subsequent working, we will assume in (3.3) that the result (3.11) can be applied, to a good approximation. For off-specular scattering, $\kappa \neq 0$, the cross-section which corresponds to (3.3) is,

$$
\frac{\mathrm{d} \sigma}{\mathrm{d} \Omega}=\left(\frac{k_{z} k_{2 z}}{\pi\left|q_{t}\right|}\right)^{2} \frac{\left(k_{z}-k_{z}^{t}\right)\left(k_{2 z}-k_{2 z}^{t}\right)}{\left(k_{z}+k_{z}^{t} *\right)\left(k_{2 z}+k_{2 z}^{t} *\right)} F\left(\kappa, q_{t}\right),
$$

where,

$$
F\left(\kappa, q_{t}\right)=\left|\int \mathrm{d} \rho \exp \left(\mathrm{i}\left(q_{t} \zeta-\kappa \cdot \rho\right)\right\}\right|^{2}
$$

and the corresponding reflectivity is obtained through (2.9). In arriving at (3.14) we have rearranged the term $\left\{V_{0} t\left(-\mathbf{k}_{2}\right) t(\mathbf{k})\right\}^{2}$ using the definitions (2.2), (2.3) and (A.3) and also utilized that, because of (3.11), all but one term in the modulus square of the 
total t-matrix element (cf. equ. (B.7)) vanish in the off-specular direction $(\kappa \neq 0)$. If both $k_{z}$ and $k_{2 z}$ are above the limit for total external reflection then (3.14) becomes

$$
\frac{\mathrm{d} \sigma}{\mathrm{d} \Omega}=\left(\frac{k_{z} k_{2 z}}{\pi q_{t}}\right)^{2} r(\mathbf{k}) r\left(-\mathbf{k}_{2}\right) F\left(\boldsymbol{\kappa}, q_{t}\right)
$$

It is convenient to develop the structure factor, $F\left(\kappa, q_{t}\right)$, using a procedure which enables it to be expressed as the Fourier transform of an autocorrelation function defined by,

$$
\left\langle\exp \left(\mathrm{i} q_{t}\left\{\zeta\left(\rho+\rho_{0}\right)-\zeta\left(\rho_{0}\right)\right\}\right)\right\rangle=\left(1 / A_{0}\right) \int \mathrm{d} \rho_{0} \exp \left(\mathrm{i} q_{t}\left\{\zeta\left(\rho+\rho_{0}\right)-\zeta\left(\rho_{0}\right)\right\}\right)
$$

This expression defines the meaning of the angular brackets, namely, a surface average as in (3.5). If the illuminated area is effectively infinite in size the reduction of $F\left(\kappa, q_{t}\right)$, defined in (3.15), to the Fourier transform of (3.17) is trivial and requires no more than a change of integration limits. While this cavalier method produces the desired result, namely, equ. (3.19), the limiting process merits some comment.

Accordingly, we note that the illuminated area may be regarded as the unit cell of a two-dimensional crystal. The phase factor $\exp \left(\mathrm{i} q_{1} \zeta\right)$ may then be expanded in a Fourier series using the appropriate reciprocal lattice. We have

$$
\exp (\mathrm{i} q, \zeta)=\sum_{\mathbf{Q}} \tau(\mathbf{Q}) \exp (\mathrm{i} \mathbf{Q} \cdot \rho)
$$

where $\mathbf{Q}$ is a reciprocal lattice vector defined by:

$$
\mathbf{Q}=m_{x}\left(\frac{2 \pi}{f}\right) \mathbf{e}_{x}+m_{y}\left(\frac{2 \pi}{h}\right) \mathbf{e}_{y}
$$

In this expression $m_{x, y}$ are integers and $\mathbf{e}_{x, y}$ are unit vectors along the axis. Thus replacing the phase factors and carrying out the integrations in (3.15) yields, 


$$
F\left(\boldsymbol{\kappa}, q_{t}\right)=A_{\circ}^{2} \sum_{\mathbf{Q}^{\prime}} \tau(\mathbf{Q}) \tau *\left(\mathbf{Q}^{\prime}\right) j_{o}\{\gamma(\mathbf{Q})\} j_{o}\left\{\gamma\left(\mathbf{Q}^{\prime}\right)\right\}
$$

where,

$$
\gamma(\mathbf{Q})=\frac{1}{2}(\mathbf{Q}-\mathbf{\kappa}) \cdot\left\{f \mathbf{e}_{x}+h \mathbf{e}_{y}\right\}
$$

If $\sqrt{A_{o}}$ is much larger than the wavelength of the probe then the sums in (3.18) are dominated by terms for which $\mathbf{Q}=\mathbf{Q}^{\prime}$. The same conditions allows us to replace the spherical Bessel functions with delta-functions using (3.13) in a manner analogous to (2.7). We find

$$
F\left(\boldsymbol{\kappa}, q_{f}\right)=4 \pi^{2} A_{o} \sum_{\mathbf{Q}}|\tau(\mathbf{Q})|^{2} \delta(\mathbf{Q}-\boldsymbol{\kappa})
$$

Invoking now the Fourier-integral representation of the delta-function and interchanging the order of summation and integration gives us

$$
F\left(\boldsymbol{\kappa}, q_{t}\right)=A_{\mathrm{o}} \int d \rho \sum_{\mathbf{Q}}|\tau(\mathbf{Q})|^{2} \exp \{\mathrm{i}(\mathbf{Q}-\mathbf{\kappa}) \cdot \rho\}
$$

Using the Fourier expansion for $\exp \left(\mathrm{i} q_{l} \zeta\right)$ it is easily established that the autocorrelation function has the expansion

$$
\sum_{\mathbf{Q}}|\tau(\mathbf{Q})|^{2} \exp (\mathrm{iQ} \cdot \rho)
$$

which gives us the desired result, namely

$$
\left.F\left(\kappa, q_{t}\right)=A_{\mathrm{o}} \int d \rho\left\langle\exp \left(\mathrm{i} q_{l} \zeta \zeta\left(\rho+\rho_{o}\right)-\zeta\left(\rho_{o}\right)\right\}\right)\right\rangle \exp \{-\mathrm{i} \kappa \cdot \rho\}
$$


In the foregoing discussion it is assumed that the area $A_{0}$ has a rectangular shape. This restriction is easily removed by dividing an arbitrary illuminated area into rectangular pieces sufficiently fine that the odd shapes adjacent to the boundary have negligible influence.

Note also that the use of the delta-functions is not formally correct since the limit is never attained in practice. Their use is, however, experimentally legitimate since they facilitate an otherwise nasty integration without appreciable errors subject, of course, to the caveat mentioned above.

Let us finally list the result for the transmission. Corresponding to (3.14) we have

$$
\frac{\mathrm{d} \sigma}{\mathrm{d} \Omega}=\left(k_{z} / 2 \pi\right)^{2} \frac{\left(k_{z}-k_{z}^{t}\right)\left(k_{2 z}^{t^{2}}-k_{2 z}^{2}\right)}{\left(k_{z}+k_{z}^{t *}\right)} G\left(\kappa, k_{z}^{t}, k_{2 z}\right),
$$

where

$$
G\left(\kappa, k_{z}^{t}, k_{2 z}\right)=A_{o} \int d \rho\left\langle\xi\left(\rho+\rho_{o}\right) \xi\left(\rho_{o}\right)\right) \exp \{-\mathrm{i} \kappa \cdot \rho\}
$$

with

$$
\xi(\rho)=\left(\exp \left\{-\mathrm{i}\left(k_{2 z}-k_{z}^{t}\right) \zeta(\rho)\right\} /\left(k_{2 z}-k_{z}^{t}\right)\right)-\left(r\left(-\mathbf{k}_{2 T}\right) \exp \left\{\mathrm{i}\left(k_{2 z}+k_{z}^{t}\right) \zeta(\rho)\right\} /\left(k_{2 z}+k_{z}^{t}\right)\right) .
$$

Observe that $k_{2 z}$ and $k_{2 z}^{t}$ refer to $\chi_{2 T}^{(-)}$specified in (A.9). The corresponding transmissivity is obtained through $(2.9)$ replacing $R$ by $T$. Note that although the expressions for the diffuse transmissivity are similar in form to the ones for the diffuse reflectivity the autocorrelation function is considerably more complicated for the transmissivity. 


\subsection{The Born approximation}

Following a similar procedure as in the previous section but. starting from $T_{21}=\left\langle\varphi_{2}|V| \varphi_{1}\right\rangle$ where $\varphi_{2}$ is a plane wave in the direction $\mathbf{k}_{2}=\left(k_{2 x}, k_{2 y}, k_{2 z}\right)$ and other quantities as earlier introduced it is readily established that the cross section for reflection in the Born approximation is,

$$
\frac{d \sigma}{d \Omega}=A_{\mathrm{o}}(N b / q)^{2} \int d \rho\left\langle\exp \left(\mathrm{i} q\left\{\zeta\left(\rho+\rho_{0}\right)-\zeta\left(\rho_{0}\right)\right\}\right)\right\rangle \exp (-\mathrm{i \kappa} \cdot \rho)
$$

where the Fermi pseudopotential, $V=\left(2 \pi \hbar^{2} / m\right) N b$, has been used and $q=k_{z}+k_{2 z}$. In the Fermi pseudopotential $m$ is the impinging particle mass and $N b$ is the scattering length density. Equ. (3.22) bears a striking resemblance to (3.14) with the insertion of (3.19), the only difference being the prefactor and that $q$ rather than $q_{t}$ appears in the autocorrelation function, and it is a trivial matter to show that $(3.14,19)$ indeed approaches $(3.22)$ in the large $k$ limit using appropriate approximations of $q_{t}$, cf. equ. (4.8). The corresponding reflectivity is as before obtained through (2.9) and in particular the specular reflectivity is conveniently derived by using a Fourier expansion of the autocorrelation function in the manner introduced in the previous section. Carrying out the $\rho$-integration in (3.22) then gives a delta-function, $\delta(\mathbf{Q}-\boldsymbol{\kappa})$, and finally integrating over an element of solid angle $\Omega$, in (2.9), sufficiently small that only $\mathbf{Q}=0$ contributes to the integral we obtain,

$$
R_{s}=(2 \pi N b / q k \sin \theta)^{2}|\tau(0)|^{2}=\left(4 \pi N b / q^{2}\right)^{2}|\langle\exp (\mathrm{i} q \zeta)\rangle|^{2}
$$

for the specular reflectivity. 


\section{Special Cases}

It is fruitful to evaluate the formulae derived in $\$ 3.2$ for some special values of the surface function $\zeta(x, y)$. The exercise will illustrate key features in the predicted crosssections, and provide results of value in the interpretation of experimental data. The cases are special in two respects; first, $\zeta(x, y)$ is independent of one co-ordinate, $y$, say, and secondly, the $x$-dependence is modelled by a simple function, which permits us to express the integrals in terms of a simple analytic function. The two cases chosen for examination are,

(a) sinusoidal grating:

$$
\zeta(x, y)=\frac{1}{2} h\left\{1-\cos \left(x p_{0}\right)\right\}
$$

and,

(b) piecewise rectangular grating:

$$
\begin{aligned}
& \zeta(x, y)=0, \quad 0 \leq x \leq d\} \text { infinite } \\
& =h, \quad d \leq x \leq \lambda\} \text { repetition. }
\end{aligned}
$$

\section{4.a Sinusoidal grating}

To evaluate the integral in (3.15) for the sinusoidal surface one can either use a standard integral representation of the Bessel function, $J_{n}(x), n=$ integer, or the standard identity,

$$
\exp (-\mathrm{i} a \cos x)=\sum_{n=-\infty}^{\infty}(-\mathrm{i})^{n} J_{n}(a) \exp (\mathrm{in} x)
$$

Applied to case (a) the parameter $a=\left(h q_{l} / 2\right)$. By either route, one quickly establishes the following result for the off-specular cross-section, 


$$
(d \sigma / d \Omega)=A_{o}\left(\frac{2 k_{z} k_{2 z}}{\left|q_{t}\right|}\right)^{2} \frac{\left(k_{z}-k_{z}^{t}\right)\left(k_{2 z}-k_{2 z}^{t}\right)}{\left(k_{z}+k_{z}^{*}\right)\left(k_{2 z}+k_{2 z}^{t} *\right)} \delta\left(\kappa_{y}\right) \sum_{n=-\infty}^{\infty}\left|J_{n}(a)\right|^{2} \delta\left(\kappa_{x}-n p_{o}\right),
$$

and,

$$
\left\langle\exp \left(\mathrm{i} q_{i} \zeta\right)\right\rangle=\exp (\mathrm{i} a) J_{0}(a)
$$

Here, it is assumed that the spatial wavelength in the models $\left(\lambda\right.$ and $\left.2 \pi / p_{0}\right)$ is sufficiently small that possible errors, due to the fact that the illuminated portion may not be an integral number of spatial wavelengths, remain negligible. This argument may be made mathematically precise by noting that the $x$-integration in (3.15) is an integral number of integrations over one wavelength plus possibly a remainder. Observe in (4.2) the presence of a selection rule which picks out multiples of the wave vector $p_{0}$.

\section{4.b Rectangular grating}

Not surprisingly, the results for the two special cases are strikingly similar. The crosssection for off-specular scattering from the rectangular grating is the same as (4.2) except that $\left|J_{n}(a)\right|^{2}$ is replaced; the explicit result is derived from the substitution,

$$
\begin{aligned}
\left|J_{\circ}(a)\right|^{2} \rightarrow & 1+\{\cos (2 a)-1\}\left(2 d(\lambda-d) / \lambda^{2}\right), \\
& \left|J_{n}(a)\right|^{2} \rightarrow(1 / \sqrt{2} n \pi)^{2}|\exp (\mathrm{i} 2 a)-1|^{2}\left\{1-\cos \left(n p_{o} d\right)\right\}, n \neq 0,
\end{aligned}
$$

where the wave number $p_{0}=(2 \pi / \lambda)$, and,

$$
\left\langle\exp \left(\mathrm{i} q_{t} \zeta\right)\right\rangle=\{d+(\lambda-d) \exp (\mathrm{i} 2 a)\} / \lambda
$$




\section{4.c Numerical studies}

To illustrate (4.2 - 4.5) and to make an important connection with optics theory it is fruitful to plot the specular and diffuse reflectivities for a few sets of parameters. We choose to study neutrons impinging on a monoatomic sample and use the Fermi pseudo-potential for $V_{0}$ in (2.2). Equ. (2.3) provides a definition of the critical edge, $k_{z c}$, for total reflection since $k_{z}^{t}$ is zero at the edge. We have,

$$
k_{z c}^{2}=k^{2}\left(1-n^{2}\right)
$$

A consequence of the Fermi pseudo-potential is that this quantity remains a constant, $k_{z c}=2 \sqrt{\pi N b}$. The modulus of $k_{z}^{t}$ therefore traces out a circle below $k_{z c}$ and a hyperbola above.

Fig. (3a) shows the specular reflectivity for the piecewise rectangular and sinusoidal models plotted against the perpendicular wave vector transfer, $q$. The set of parameters have been chosen for the sake of clarity rather than to represent an actual physical system or experiment. For comparison the reflectivity from an optical flat is also shown. The most striking features are the interference fringes. Their mathematical origin is clear from (4.3) and (4.5) and they may be physically understood from the fact that as far as the specular reflection is concerned the system behaves as a smooth bulk with a thin film on top. The fringes for the rectangular grating touch the curve for the optical flat as opposed to that for the sinusoidal. This is because the mimicked thin film has a constant refractive index in the former case whereas it is a continuously varying function of $z$ in the latter. Note also the models' departure from physically plausible values as the critical edge is approached from the right. 
The dissimilarity below the critical edge stems from the different forms attained in this region, i.e.

$$
\exp (\mathrm{i} a) J_{0}(a) \rightarrow \exp (-|a|) I_{0}(|a|)
$$

and,

$$
\exp (\mathrm{i} 2 a) \rightarrow \exp (-2|a|)
$$

where $I_{0}$ is the zeroth order modified Bessel function of the first kind. Fig (3.b) shows the effect of increasing $h$ and decreasing $N b$, namely that the critical region shrinks and the fringes squeeze. The set of parameters in this case is representative of an experiment performed on nickel provided that resolution is infinite.

Fig. (4) shows the influence of $d / \lambda$ for the rectangular grating. The amplitude of the fringes decreases if $d / \lambda$ is changed from 0.5 , for which it attains a maximum, as expected, and also the point where the model leaves the physical world is shifted. The fact that a thin film is mimicked makes it interesting to compare our results with those derived from standard optics theory, and for the rectangular grating this is particularly simple to do. In this case the refractive index for the 'film' is a constant, as has already been pointed out, and this allows the use of Airy's formula [6] describing the reflectivity including infinite summation of multiple reflections. We have:

$$
r=\frac{r_{12}+r_{23} \exp (\mathrm{i} \delta)}{1+r_{12} r_{23} \exp (\mathrm{i} \delta)}
$$

where $r_{12}$ is the Fresnel coefficient for radiation incident from vacuum/air onto the film, $r_{23}$ is the corresponding quantity for incidence from the film onto the bulk and $\delta$ is the optical path 
difference between two successive reflections. For the rectangular grating we have the following refractive indices,

$$
\begin{aligned}
& n_{\text {film }}=\left(1-\frac{4 \pi N b}{k^{2}} \frac{d}{\lambda}\right)^{1 / 2} \\
& n_{\text {bulk }}=\left(1-\frac{4 \pi N b}{k^{2}}\right)^{1 / 2}
\end{aligned}
$$

and

$$
\delta=2 k_{z} h n_{\mathrm{film}}
$$

The various $z$-components in $r_{12}$ and $r_{23}$ may be obtained using Snell's law of refraction or more conviniently from (4.6) and (2.3) using appropriate critical edges for the film and the bulk. Fig. (5) shows the resulting reflectivity and that obtained from the distorted wave Born approximation (DWBA). The excellent agreement not too close to or within the critical region is not coincidential. In fact, the the two expressions have the same asymptotic limit for large $q$, which is readily demonstrated by firstly writing (2.3) in the form,

$$
k_{z}^{\prime}=\left(k_{z}^{2}-k_{z c}^{2}\right)^{1 / 2} \sim k_{z}-\frac{1}{2} \frac{k_{z c}^{2}}{k_{z}}
$$

which after some manipulation leads to,

$$
r \sim \frac{k_{z c}^{2}}{4 k_{z}^{2}}=(\pi N b) / k_{z}^{2}
$$

or 


$$
R \sim(\pi N b)^{2} / k_{z}^{4}
$$

for the Fresnel reflectivity (equ. (A.4)) and secondly by inserting corresponding approximations into the asymptotic expressions which are:

$$
\begin{aligned}
& R_{\mathrm{DWBA}} \sim R\left\{d^{2}+(\lambda-d)^{2}+2 d(\lambda-d) \cos \left(2 k_{z}^{t} h\right)\right\} / \lambda^{2} \\
& R_{\text {Airy }} \sim R_{12}+R_{23}+2 \operatorname{Re}\left\{r_{12} r_{23}^{*} \exp (-\mathrm{i} \delta)\right\} .
\end{aligned}
$$

In the same limit, $2 k_{z}{ }^{t} h=\delta$ which completes the proof. The result is a manifestation of the fact that both the DWBA and the Airy formula tend to the Born approximation for large incident energies. It furthermore demonstrates that, plausible values are obtained also for large deviations implying that the same would hold for off-specular scattering. It appears then, perhaps surprisingly, that in the present perturbation theory the magnitude of the deviations is not a sufficient smallness parameter. Rather, the quality of the DWBA depends on the extent to which the Fresnel solutions correctly describe the scattered waves which indeed they seem to do remarkably well, except, of course, near and in the critical region.

Turning now to the diffuse reflectivity figs. (6a) and (6b) show the first order reflections for the rectangular and sinusoidal gratings plotted against $2 k_{z}$ which is equal to $q$ used for the specular reflectivity. Positive orders correspond to scattering angles, $\theta_{2}$, less than $\theta$, which characterises the specular direction and negative orders to $\theta_{2}>\theta$. The 'sinusoidal' reflectivities are generally smaller than the 'rectangular' ones as was also the case for the specular direction. It is also worth noting that the dips actually reach zero ( equs. (4.2) and (4.4) ) but the data is too sparse to show it. We also note that the 'positive' fringes are shifted relative to the 'negative' ones. This stems from the behaviour of $q_{t}=k_{z}^{t}+k_{2 z}{ }^{t}$ where $k_{2 z}{ }^{t}$ is larger on the positive side than on the negative. Around $2 k_{z}=0.5 \AA^{-1}$ there are two peaks for 
each curve caused by a transition between different analytical forms as either $k_{z}$ or $k_{2 z}$ passes the critical value. Plotted in these figures are also the scattering angles, given by,

$$
\cos \theta_{2}=\cos \theta+\kappa_{x} / k
$$

as is obvious from geometrical considerations. It is seen that these asymptotically approach the specular direction as the incident energy is increased. Since $\kappa_{x}$ is a constant for each order this follows from (4.9). There exists also a minimum $k_{z}$ for which a particular order first appears and this is set by $\theta_{2}=0$ for the positive side and by $\theta_{2}=\pi$ for the negative. This minimum increases with order and is larger for the positive orders than for the negative.

Fig. (7) shows second order reflectivities for the sinusoidal grating. Even orders vanish for the rectanglar if $d / \lambda=0.5$ as is obvious from (4.2) where the factor in curly brackets is zero for even $n$. Figs. ( $8 \mathrm{a})$ and ( $8 \mathrm{~b})$ shows the third order demonstrating that the sinusoidal grating 'misses' the first dip. The trend is systematic; for the fifth order the first two dips would be 'missed' and so on. This is because the first zero of the Bessel function is sensitive to the order as well as to the actual argument. Note, finally, that the peak value for each fringe systematically decreases with the order magnitude as expected from optics theory on gratings which dictates that higher orders are more dispersive and less intense.

\section{Random Surface Structures}

Let us enquire about the features of scattering by a rough surface that is legitimately described in terms of a stochastic distribution. To be more precise, the distribution in question defines the properties of $\zeta(\rho)$ treated as a stochastic variable. The distribution in principle completely defines $\zeta(\rho)$ and all its correlation functions. For our definition of the surface function, depicted in fig. (2), the average value of $\zeta$ is not zero. In keeping with our previous notation, the average value of a variable with respect to the distribution is denoted by angular brackets. So, the foregoing remark about the surface function amounts to $\langle\zeta\rangle \neq 0$. Averages of higher powers of $\zeta$ enter the subsequent discussion, e.g. the so-called variance, 


$$
\sigma^{2}=\left\langle(\zeta-\langle\zeta\rangle)^{2}\right\rangle
$$

The lowest-order spatial correlations are conveniently handled in terms of an autocorrelation function $C(\rho)$ defined by,

$$
\sigma^{2} C(\rho)=\left\langle\zeta\left(\rho+\rho_{0}\right) \zeta\left(\rho_{o}\right)\right\rangle-\langle\zeta\rangle^{2}
$$

where $\rho_{0}$ is a dummy variable as in (3.17). Here, we have assumed that the distribution is independent of the reference frame, i.e. the surface is uniform (homogeneous) on a microscopic scale. Spatial inversion symmetry, which will arise from isotropy found in a fluid or the high symmetry of the point group of simple crystal structures, dictates that $C(\rho)$ is independent of the orientation of the vector $\rho$; for this special case the autocorrelation function is written as $C(\rho)$. By definition, $C(0)=1$, and $|C(\rho)| \leq 1$.

Having dispatched these necessary comments and definitions about random surface structures, let us turn to the formulation of the scattering. We elect to treat first specular scattering. The starting point is (3.5). A cumulant expansion is a useful scheme to handle the average of $\exp \left(\mathrm{i} q_{t} \zeta\right)$. To order $q_{t}^{3}$ in the argument of the exponential function,

$$
1-\langle S\rangle=\exp \left\{\mathrm{i} q_{t}\langle\zeta\rangle-\frac{1}{2} q_{t}^{2}\left(\left\langle\zeta^{2}\right\rangle-\langle\zeta\rangle^{2}\right)-\frac{1}{6} q_{t}^{3}\left(\left\langle\zeta^{3}\right\rangle-3\left\langle\zeta^{2}\right\rangle\langle\zeta\rangle+2\langle\zeta\rangle^{3}\right)-\cdots\right\}
$$

Higher-order terms in the cumulant expansion are readily derived. For the special case of a simple Gaussian model distribution, the coefficients in (5.3) of $q_{?}^{3}$ and all higher powers vanish leaving the estimate,

$$
\langle S\rangle \sim 1-\exp \left\{\mathrm{i} q_{t}\langle\zeta\rangle-\frac{1}{2}\left(\sigma q_{t}\right)^{2}\right\}
$$

The corresponding reflectivities are obtained from (3.7), and (5.3) or (5.4); for the Gaussian model distribution above the critical edge,

$$
\begin{aligned}
R_{\mathrm{s}}=R\left\{\left(1-k_{z} / k_{z}^{t}\right)^{2}\right. & +2\left(1-k_{z} / k_{z}^{t}\right)\left(k_{z} / k_{z}^{t}\right) \exp \left(-\frac{1}{2}\left(\sigma q_{t}\right)^{2}\right) \cos \left(q_{t}\langle\zeta\rangle\right) \\
& \left.+\left(k_{z} / k_{z}^{t}\right)^{2} \exp \left(-\left(\sigma q_{t}\right)^{2}\right)\right\} .
\end{aligned}
$$


Note that with our definition of $\zeta$ its mean value is actually infinite in the context of the Gaussian model. The physically significant reflectivity is therefore the appropriate limit of the expression above. In physical terms the interference fringes collapse on top of each other and the corresponding term must therefore vanish, leaving,

$$
R_{\mathrm{s}}=R\left\{\left(1-k_{z} / k_{z}^{t}\right)^{2}+\left(k_{z} / k_{z}^{t}\right)^{2} \exp \left(-\left(\sigma q_{t}\right)^{2}\right)\right\}
$$

The surface average calculation together with the physical argument just presented is known to mathematicians as an application of the Riemann - Lebesgue theorem, which may be found in elementary texts on Fourier analysis. In this context, it must be pointed out that any real surface of course is limited in the extent of $\zeta$ and therefore should exhibit interference. The correct thing to do is then to truncate the Gaussian distribution but in most circumstances the benefits of so doing are modest in comparison with just using (5.5). In this discussion, we are not primarily concerned with the behaviour of the interference fringes since they, to our knowledge, have not been observed. It is, however, interesting to note that the bottom envelope of (5.5) reaches zero when $1-\exp \left(-\frac{1}{2}\left(\sigma q_{t}\right)^{2}\right)=k_{z}^{i} / k_{z}$ and at this point there is a maximum in the amplitude of the fringes. This is shown in fig. (9) in which both (5.5) and its envelopes are plotted for a set of parameters such that the mean value of $\zeta$ is twenty times the mean square deviation. Again, we have used the Fermi pseudo-potential for neutrons impinging on a monoatomic sample.

Returning to (5.6) we note that with $k_{z}^{t}$ given by the Fermi pseudopotential $k_{z}^{t} \rightarrow k_{z}$ $\left(q_{t} \rightarrow q\right)$ as $k \rightarrow \infty$ and it would appear that (5.6) approaches the Born approximation, equ. (3.23) in this limit. This is, however, not the case as is shown in fig. (10). In view of the result for the piecewise rectangular grating and also quite generally from the fact that, within the framework of the Fermi pseudopotential, the t-matrix element in the distorted wave Born approximation asymptotically approaches that of the Born approximation in the large $k$ limit the result appears somewhat alarming, and one is tempted to believe that there is actually something wrong with the derivation. The fact remains, though, that it is by the properties of the distribution of $\zeta$ the result comes about. This may be realized by noting that, the surface average of $\exp \left(\mathrm{i} q_{i} \zeta\right)$ essentially gives the Fourier transform of the distribution and thereby 
determines the $q_{t}$ dependence. In other words, for the Gaussian model the distorted wave functions simply do not go to plane waves sufficiently fast in the large $k$ limit to retain the Born approximation. It would appear that, for the Gaussian model $\sigma$ is a proper smallness parameter in the perturbation. Since the perturbation theory has been terminated at the first level it is conceivable that the apparent difficulties with the model could be resolved by going to higher order perturbation. Note, though, that the large $k$-problem only exists in the specular direction.

Off-specular scattering is slightly more algebraically complicated. The quantity of central interest in this instance is the structure factor,

$$
F\left(\kappa, q_{t}\right)=A_{0} \int \mathrm{d} \rho \exp (-\mathrm{i} \kappa \cdot \rho)\left\{\left\langle\exp \left(\mathrm{i} q_{t}\left[\zeta\left(\rho+\rho_{0}\right)-\zeta\left(\rho_{0}\right)\right]\right)\right\rangle-\mid\left\langle\exp \left(\mathrm{i} q_{t} \zeta\left(\rho_{0}\right)\right)\right\rangle^{2}\right\}
$$

We have subtracted a term proportional to $\delta(\kappa)$ which is zero for off-specular scattering $(\kappa \neq 0)$, to arrive at a form that is convenient to use in the numerical evaluation of the integral.

In general, one can choose to write,

$$
\left\langle\exp \left(\mathrm{i} q_{t}\left(\zeta-\zeta^{\prime}\right)\right)\right\rangle=\int \mathrm{d} \zeta \mathrm{d} \zeta^{\prime} W\left(\zeta, \zeta^{\prime}\right) \exp \left(\mathrm{i} q_{t}\left(\zeta-\zeta^{\prime}\right)\right)
$$

where $W\left(\zeta, \zeta^{\prime}\right)$ is a normalized joint probability function. Observe that (5.8) is the generating function for correlation functions formed with powers of $\left(\zeta-\zeta^{\prime}\right) \equiv\left\{\zeta(\rho)-\zeta\left(\rho^{\prime}\right)\right\}$, e.g. $C(\rho)$ in (5.2).

By way of an illustration, let us go on and consider a Gaussian model, namely,

$$
W\left(\zeta, \zeta^{\prime}\right)=\left\{f_{\mathrm{o}}\left(1-C^{2}\right)^{1 / 2} / \pi\right\} \exp \left\{-f_{\mathrm{o}}\left(\zeta^{2}+\zeta^{\prime 2}-2 \zeta \zeta^{\prime} C\right)\right\}
$$

Here, $C=C(\rho)$ is defined in (5.2) and,

$$
f_{0}(\rho)=\left\{2 \sigma^{2}\left(1-C^{2}(\rho)\right)\right\}^{-1}
$$

with $\sigma^{2}$ as in (5.1). The corresponding estimate for the structure factor is, 


$$
F\left(\kappa, q_{t}\right)=A_{o} \int d \rho \exp (-\mathrm{i} \kappa \cdot \rho)\{g(\rho)-g(\infty)\}
$$

where,

$$
g(\rho)=\exp \left\{-\left(\sigma q_{t}\right)^{2}[1-C(\rho)]\right\}
$$

and in the limit $\rho \rightarrow \infty$,

$$
g(\infty)=\exp \left(-\left(\sigma q_{t}\right)^{2}\right)
$$

Of course, the result for (5.9) which we have used to obtain (5.11) is consistent with the definition $(5: 2)$ of $C(\rho)$. In the event that the surface structure is spatially isotropic the autocorrelation function in $g(\rho)$ is independent of the orientation of $\rho(5.11)$ reduces to,

$$
\begin{aligned}
F\left(\kappa, q_{t}\right)=\pi A_{0} \int_{0}^{\infty} \mathrm{d} \rho^{2} J_{0}(\kappa \rho)\{g(\rho)-g(\infty)\} \\
\quad=\pi A_{0} \int_{0}^{\infty} \mathrm{d} \rho^{2}\left\{1-(\kappa \rho)^{2} / 2+\cdots\right\}\{g(\rho)-g(\infty)\},
\end{aligned}
$$

where the last line is appropriate for the consideration of the limit $\kappa \rightarrow 0$.

It is interesting to note that, in the limit $\sigma q_{t}<<1$ we get to order $\left(\sigma q_{t}\right)^{2}$,

$$
F\left(\kappa, q_{t}\right)=\pi A_{0}\left(\sigma q_{i}\right)^{2} \int_{0}^{\infty} \mathrm{d} \rho^{2} J_{0}(\kappa \rho) C(\rho)
$$

i.e. the off-specular scattering is directly proportional to a Bessel transform of the autocorrelation function, $C(\rho)$. (If the surface structure is not spatially isotropic the twodimensional Fourier transform replaces the Bessel transform in (5.14).) In Table 1 we list a few such transforms of physical interest. Note in particular that if $C(\rho)$ is a Gaussian then so is the transform. On a plane perpendicular to the specular direction the isoreflectivities for isotropic surfaces will be ellipses with the eccentricity set by the angle of incidence as is evident from geometrical considerations. It is thus sufficient to illustrate the reflectivity for one azimuth, say 0 , which corresponds to the plane defined by the incident beam and the mean surface normal. In fig. (11) we show the reflectivity for a surface with a Gaussian autocorrelation function, labelled (1) in Table 1. The peak at $\theta_{2} \sim 1^{\circ}$ corresponds to the so 
called Yoneda scattering [7] and stems from the prefactor in (3.14). We see that the offspecular scattering as expected increases if the correlation length increases.

Another example from Table 1 also merits comment, namely-number (4). In this example $C(\rho)$ contains a oscillatory function, $J_{0}(2 \rho / \lambda)$, where $\lambda$ may be regarded as the wavelength. This feature is reminiscent of the periodic gratings considered in $\$ 4$ and this makes it interesting to compare with the Bragg scattering resulting from such structures. In this case the possibility of one off-specular peak arises as the result of the competition between the modified Bessel function, $I_{0}$, and the Gaussian in the transform. The interplay between the parameters, $\lambda$ and $\xi$, may be described as follows. If $(\xi / \lambda)$ is small the Gaussian term in $C(\rho)$ dominates and off-specular scattering increases as $\xi$ increases until oscillations in $J_{0}$ start to exert an appreciable influence. At a general angle this causes off-specular scattering to decrease but for particular values of $\xi$ and $\lambda$ an off-specular peak results.

With care this peak can be brought within the plotted range of $\theta_{2}$ which corresponds to $\left(\sigma q_{t}\right) \leq 0.3$ as demonstrated in fig. (12). Even though the off-specular peak cannot legitimately be related to Bragg scattering the effect of the oscillations yields constructive interference in an off-specular direction. Note a difference of about 8 orders of magnitude between the peak height and its neighbourhood.

\section{Discussion and Conclusions}

Our formulation of the distorted wave Born approximation applied to scattering by rough surfaces provides a consistent level of approximation for both specular and off-specular scattering, in contrast to the formulations found in the works by Sinha et al. [8] and Pynn [4]. In those works the authors study Gaussian surfaces, and choose the mean surface to be the reference surface which requires them to introduce an analytical continuation of the wave functions in order to obtain convenient results for off-specular scattering. Our result for the off-specular scattering is, however, equivalent to Sinha's et al [8], and almost the same as Pynn's [4] result. This conclusion stems from the fact that the result for off-specular scattering does not depend on the choice of reference surface. The main advantage of the present work is a compact result derived without having to introduce an additional raft of approximations.

With regard to specular scattering, our result is considerably less complicated than Sinha's et al [8] corresponding expression and consequently easier to interpret. Pynn [4], on 
the other hand, uses analytical continuation and a somewhat different wave function which lead him to a result previously presented by Nevot and Croce [9]. The present result, however, does not connect very easily to the work by Nevot and Croce [9], namely

$$
R_{\mathrm{s}}=R \exp \left(-\sigma^{2} q q_{t}\right)
$$

Only if $\sigma q_{t}<<1$, and $q \sim q_{t}$ i.e. large $q$, (equ. 5.6) is consistency obtained. But, needless to say, the two conditions are for practical purposes almost incompatible. The inadequacy of the distorted wave Born approximation below the critical edge is mentioned by Sinha et al., who also attribute the deficiency to an early truncation of the Born series. In view of this deficiency, the behaviour below the critical edge of our result ( and those of Sinha et al. and Pynn ) for off-specular scattering appears to be questionable even though unphysical values are not evident in our calculations.

Staying with the Gaussian model, it is worth pointing out that it does not suffice to regard, as has been done, the two reference points in the autocorrelation function as independent variables for this requires that $C(\rho)=0$ for $\rho \neq 0$. In other words, if two independent stochastic variables have Gaussian distributions they are uncorrelated. If the autocorrelation function is pushed to such a limit, i.e. $C(\rho) \propto \delta(\rho)$, the corresponding surface only exhibits specular reflection, and it is clear that such a surface can no longer be regarded as an analytical surface in the traditional sense, i.e. a piecewise continuous bounded function, but must be something else, presumably a surface with a graded refractive index.

Quite generally, the diffuse scattering cross-section is proportional to the Fourier transform of the autocorrelation function of the phase factors caused by the deviations, $\zeta$, from the reference surface (equs. 3.14 and 3.19). The proportionality constant is easily determined from the experimental geometry, and a knowledge of the incident energy. This makes it tempting to propose an inversion scheme. From signal analysis we know that, the Fourier transform of the autocorrelation function is the same as the squared modulus of the spectrum which would be the Fourier transform of the phase shifts themselves. An inverse Fourier transform of the square root would therefore give $\zeta(\rho)$ to within an integral number of wavelengths at each wavelength. The square root is, however, not uniquely given and this problem is brought about by a loss of phase information. This information is theoretically retrievable by using the Kramers-Kronig relations but it assumes that the cross-section is known for all frequencies which is never true in practice. In addition, the off-specular cross- 
section for random structures generally falls so rapidly to zero that truncation of the original Fourier transform would be necessary. The resulting inverse Fourier transform would then be contaminated by convolutions with spherical Bessel functions. There are ways to deal with such contaminations but in view of all other limitations it appears that a reconstruction of the surface stands little chance of success. The conclusion is therefore that, comparison between simulations based on model surfaces for the present is the best way of assessing surface structures as has been previously pointed out [1].

The connection of our findings to the Born approximation (BA) is simple in the offspecular case as the distorted wave Born approximation asymptotically approaches the BA in the large $q$ limit. In the specular case it is not so simple and whether the BA is approached or not depends on the distribution of the deviations. For periodic structures the distribution is peaked at several points which appears to be a sufficient condition to retain the BA. For nonperiodic Gaussian structures the distribution is peaked only at the mean surface, a behaviour which causes a too fast $q_{t}$-dependence through the surface average for the BA to be retained. Furthermore, $\sigma$ as defined by (5.1) and applied to the periodic structures does not seem to be a sufficient smallness parameter in the perturbation whereas this is indeed the case for the Gaussian model.

Our results have been derived using incident plane waves which have infinite coherence lengths. Needless to say, such waves do not exist in reality and whether predicted interference structure is observed depends, of course, on the degree of coherence in the incident beam. The finer the interference structure is, the less observable it becomes. In Appendix $\mathrm{C}$ we briefly comment on and estimate the relevant coherence lengths in an actual experiment.

Finally, let us point out that, in principle, there is no sharp boundary between specular and off-specular scattering. Rather, because of Fraunhofer diffraction the specular scattering is distributed over the entire hemisphere although most of it is concentrated in a small solid angle in the specular direction (the larger the illuminated area (aperture) the smaller the solid angle). The off-specular scattering is likewise distributed for the same reason. The results given in the previous sections rest on the assumption that the influence from the limited aperture is negligible which is certainly true if the illuminated area is large compared to the particle wavelength. 


\section{Acknowledgements}

We are grateful to a number of scientists, for their interest and inputs to our work, including I. Anderson, J. Penfold, R. Pynn, H. Rauch, P. Schofield, O. Schärpf, E. G. Staples, B. Toperverg and J. R. P. Webster.

One of us (G.L.) has received financial support form the E.C., H.C.M. programme contract ERBCHB1CT920068. 


\section{References}

1. Vineyard, G. H., Phys. Rev. B26, 4146 (1982).

2. Dosch, H., "Critical Phenomena at Surfaces and Interfaces" (Springler-Verlag, N.Y. 1992).

3. Dietrich, S. and Wagner, H., Z. Phys. B59, 35 (1985).

4. Pynn, R., Phys. Rev. B45, 602 (1992).

5. Sears, V.F., "Neutron Optics" (O.U.P., Oxford, 1989).

6. Born, M. and Wolf, E., "Principles of Optics” (Pergammon Press, N.Y. 1975).

7. Yoneda, Y., Phys. Rev. 131, 2010 (1963).

8. Sinha, S. K., Sirota, E. B., Garott, S. and Stanley, H.B., Phys. Rev. B38(4), 2297 (1988).

9. Nevot L. and Croce P., Rev. Phys. Appl. 15, 761 (1980).

10. Newton, R. G., "Scattering Theory of Waves and Particles" (Springer-Verlag, N.Y. 1982). 


\section{Appendix A}

\section{Reflection and Refraction by an Optical Flat.}

Referring to fig. (1), the $x-y$ plane is here taken to define a perfectly flat plane that separates a vacuum half-space from a medium $(z<0)$. The plane waves which are incident, reflected and refracted have wave vectors $\mathbf{k}, \mathbf{k}^{\prime}$ and $\mathbf{k}^{t}$, respectively. The magnitudes of these wave vectors satisfy $k=k^{\prime}$, and $k^{b}=n k$ where $n$ is the refractive index in the medium. (Total external reflection can occur if $n<1$ ). If the wave vectors of the incident and reflected waves make an angle $\theta$ with $x-y$ plane, as depicted in fig. (1), then the $z$-components of $\mathbf{k}$ and $\mathbf{k}^{t}$ satisfy,

$$
n^{2}=\left(k^{t} / k\right)^{2}=\cos ^{2} \theta+\left(k_{z}^{t} / k_{z}\right)^{2} \sin ^{2} \theta
$$

or,

$$
\left(k_{z}^{t} / k_{z}\right)=\left\{1-\left(1-n^{2}\right) / \sin ^{2} \theta\right\}^{1 / 2},
$$

and since $k_{z}=k \sin \theta$,

$$
k^{2}\left(1-n^{2}\right)=k_{z}^{2}-\left(k_{z}^{t}\right)^{2}
$$

These relations stem from the application of Cauchy boundary conditions to the wave function at the $x$ - $y$ plane, namely continuity at $z=0$ of both the wave function and its spatial derivative normal to the surface.

The Fresnel wave functions for a plane surface are,

$$
\chi(\mathbf{r})=\left\{\begin{array}{c}
\exp (\mathrm{ik} \cdot \mathbf{r})+r(\mathbf{k}) \exp \left(\mathbf{i k}^{\prime} \cdot \mathbf{r}\right) ; \quad z \geq 0 \\
t(\mathbf{k}) \exp \left(\mathrm{ik}^{t} \cdot \mathbf{r}\right) ; \quad z \leq 0
\end{array}\right.
$$


The components of the wave vectors for the incident, reflected and refracted waves satisfy $k_{z}^{\prime}=-k_{z}$, and $k_{\alpha}=k_{\alpha}^{\prime}=k_{\alpha}^{t}$ with $\alpha=x$ or $y$, and we will choose $\mathbf{k}=\left(k_{x}, 0,-k_{z}\right), \mathbf{k}^{\prime}=\left(k_{x}, 0, k_{z}\right)$ and $\mathbf{k}^{t}=\left(k_{x}, 0,-k_{z}^{t}\right)$, c.f. fig. (1). The Cauchy boundary conditions applied to $\chi(\mathbf{r})$ at $z=0$ lead to the relations,

$$
1+r=t, \quad \text { and }-k_{z}+k_{z} r=-k_{z}^{t} t
$$

or,

$$
r(\mathbf{k})=\left(k_{z}-k_{z}^{t}\right) /\left(k_{z}+k_{z}^{t}\right), \text { and } t(\mathbf{k})=2 k_{z} /\left(k_{z}+k_{z}^{t}\right)
$$

with $k_{z}^{t}$ determined via (A.1) from $\mathbf{k}$. The coefficient $r(\mathbf{k})$ and $t(\mathbf{k})$ enter also the expression for the conservation of radiation flux across the plane surface. A separate calculation shows that the reflectivity, $R(\mathbf{k})$, and transmissivity, $T(\mathbf{k})$, are [5],

$$
R(\mathbf{k})=|r(\mathbf{k})|^{2}
$$

and,

$$
T(\mathbf{k})=\left(k_{z}^{t} / k_{z}\right) t^{2}(\mathbf{k})
$$

and flux conservation is manifest in the relation,

$$
R(\mathbf{k})+T(\mathbf{k})=1
$$

Total external reflection occurs for parameters such that $R(\mathbf{k})=1$. If the refractive index is less than unity, inspection of (A.1) shows that the reflectivity is unity for small grazing angles less than $\sqrt{ }\left(1-n^{2}\right)$.

The foregoing results appertain to a smooth surface. If, on the other hand, the surface is rough then off-specular transmission and reflection occur with corresponding 
modifications of $T$ and $R$. As a given surface may appear rough or smooth depending on the angle of incidence it is not sufficient to consider the deviations from a reference surface alone, rather, the surface is conventionally considered smooth if,

$$
2 k\left\langle(\zeta-<\zeta>)^{2}\right)^{1 / 2} \sin \theta<(\pi / 4)
$$

otherwise it is rough. The angular brackets denote an average over the surface, $\zeta=\zeta(x, y)$ as defined in fig. (2) in the main text and $\theta$ is the angle of incidence measured from the reference plane. Equ. (A.6) is a formulation of the so-called Rayleigh criterion. In the main text the roughness influence is estimated using the distorted wave Born approximation outlined in Appendix B.

It remains here to define the various wavefunctions appearing in (B.7) and (B.11), namely $\chi_{1}^{(+)}$and $\chi_{2}^{(-)}$. As distorting potential that of an optical flat is used. The scattered field consists therefore of a reflected beam in the upper hemisphere and a transmitted one in the lower. As the wave function is the sum of incident and scattered fields we have $\chi_{1}^{(+)}=\chi(\mathbf{r})$ as specified in (A.2). The ${ }^{(+)}$indicates that the scattered waves are of outgoing type, which brings us to the ${ }^{(-)}$appearing in $\chi_{2}^{(-)}$. In this case the scattered waves have to be of incoming type i.e. the 'reflected' and 'transmitted' beams meet at the interface and the 'incident' leaves it. Such a wave function is specified as follows:

$$
\chi_{2}^{(-)}(\mathbf{r})=\left\{\begin{array}{c}
\exp \left(\mathbf{i k}_{2} \cdot \mathbf{r}\right)+r^{*}\left(-\mathbf{k}_{2}\right) \exp \left(\mathbf{i} \mathbf{k}_{2}^{\prime} \cdot \mathbf{r}\right) ; z \geq 0 \\
t^{*}\left(-\mathbf{k}_{2}\right) \exp \left(\mathrm{ik}_{2}^{t} \cdot \mathbf{r}\right) ; z \leq 0,
\end{array}\right.
$$

where $\mathbf{k}_{2}=\left(k_{2 x}, k_{2 y}, k_{2 z}\right), \mathbf{k}_{2}^{\prime}=\left(k_{2 x}, k_{2 y},-k_{2 z}\right)$ and $\mathbf{k}_{2}^{t}=\left(k_{2 x}, k_{2 y}, k_{2 z}^{t}\right)$.

A wave function of the kind above is often referred to as a time reversed wave function because of its backward behaviour. To fully appreciate its name and formal properties it is necessary to consider the time aspect which up to now has been hidden 
in an omitted phase factor $\exp (-\mathrm{i} \omega t)$. As a scattering event of necessity is time dependent the wave functions above only represent steady state solutions in which a steady stream of particles are being continuously scattered. For the single scattering event we have to consider the time dependent Schrödinger equation,

$$
\mathscr{H} \Psi=\mathrm{i} \hbar \frac{\partial}{\partial t} \Psi
$$

By taking the complex conjugate on both sides we find (since $\mathscr{H}$ is real):

$$
\Re \Psi *=-\mathrm{i} \hbar \frac{\partial}{\partial t} \Psi *=i \hbar \frac{\partial}{\partial(-t)} \Psi *
$$

Thus, if $\phi=\Psi *(-t)$ then $\phi$ also satisfies (A.8) and is called the time reversed solution corresponding to $\Psi$. So we see that, in order to form a time reversed solution from a known one, we have to make a complex conjugation and change the sign of the time variable. Since the time dependence is specified by the phase factor $\exp (-i \omega t)$ this remains unchanged by the operation and it may therefore still be omitted. All we need care about is therefore the complex conjugation which is partly made implicit by the definition of $\mathbf{k}_{2}, \mathbf{k}_{2}^{\prime}$ and $\mathbf{k}_{2}^{t}$.

The wave function (A.7) represents in the infinite future the scattered wave $\exp \left(\mathbf{i k}_{2} \cdot \mathbf{r}\right)$ emerging in the upper hemisphere and (A.8) is therefore the appropriate wave function in the consideration of specular and diffuse reflection but it will not do for transmission. In this instance yet another time reversed wave function must be used, namely,

$$
\chi_{2 T}^{(-)}(\mathbf{r})=\left\{\begin{array}{c}
t *\left(-\mathbf{k}_{2 T}\right) \exp \left(\mathrm{ir} \cdot \mathbf{k}_{2 T}^{t}\right) ; z \geq 0 \\
\exp \left(\mathrm{ik}_{2 T} \cdot \mathbf{r}\right)+r *\left(-\mathbf{k}_{2 T}\right) \exp \left(\mathrm{ik}_{2 T}^{\prime} \cdot \mathbf{r}\right) ; z \leq 0
\end{array}\right.
$$


where $\mathbf{k}_{2 T}=\left(\mathbf{k}_{2 x}, \mathrm{k}_{2 y},-\mathrm{k}_{2 z}\right), \mathbf{k}_{2 T}^{\prime}=\left(k_{2 x}, k_{2 y}, k_{2 z}\right)$ and $\mathbf{k}_{2 T}^{t}=\left(k_{2 x}, k_{2 y}, k_{2 z}^{t}\right)$. In the infinite future this wave function represents the scattered wave $\exp \left(i_{k_{2 T}} \mathbf{r}\right)$ emerging in the lower hemisphere.

The wavefunctions $\chi_{1}^{(+)}, \chi_{2}^{(-)}$and $\chi_{2 T}^{(-)}$are depicted in fig. (13) where the, in theory, infinitely wide beams are represented by arrows, which are parallel to the propagation vectors. Observe that, since the incident beam is destroyed and replaced by the reflected and transmitted beams due, to the multiple scattering embodied in the refractive index, there is never any danger of contaminating the detected signal with incident particles. 


\section{Appendix B}

\section{Distorted Wave Formulation of Scattering Theory}

Our goal is to précis scattering theory with a view to providing some insight to the distorted wave formulation and the approximate scattering theory used in the main text. A full and rigorous account of scattering theory is provided, for example, by Newton [10]. Perhaps it is worth mentioning that the distorted wave formulation was developed in the context of the interpretation of charged particle scattering by nuclei. In this case, the Coulomb part of the scattering potential defines a problem that can be solved without approximation. Hence, a scheme which suggests itself is to treat the non-Coulomb part of the potential as a perturbation and develop an expansion in which matrix elements of the perturbation are calculated with the (known) wave functions for the Coulomb potential problem.

Let us start by recapitulating the elements of the time-independent formulation of scattering theory. If $\mathcal{H}_{0}$ and $V$ denote a Hamiltonian and an interaction potential, respectively, then the wave functions $\psi$ and $\varphi$ in the Schrödinger equations,

$$
\left(E-H_{0}\right) \psi=V \psi
$$

and,

$$
\left(E-\mathscr{H}_{0}\right) \varphi=0,
$$

are related by the Lippmann-Schwinger equation,

$$
\psi=\varphi+\left(E-\mathcal{H}_{0}\right)^{-1} \quad V \psi
$$

The appropriate boundary conditions can be stipulated by adding an infinitesimal imaginary part to the energy variable, $E$. On making the substitution $E \rightarrow E \pm$ in with 
$\eta=0^{+}$the plus (minus) sign in the substitution selects an outgoing (incoming) wave. The corresponding wave functions are denoted by $\psi^{( \pm)}$. Turning next to the crosssection for scattering by $V$, into an element of solid angle $\mathrm{d} \Omega$, let the labels " 1 " and " 2 " refer to the initial and final states, respectively. The wave functions $\varphi_{1}$ and $\varphi_{2}$ are the wave functions of the parts of the system at an infinite distance apart, which is a situation in which $V$ exerts no influence. The cross-section, $(\mathrm{d} \sigma / \mathrm{d} \Omega)$, is obtained from the asymptotic form of the wave function, namely,

$$
-\left(m / 2 \pi \hbar^{2}\right)<\varphi_{2}|T| \varphi_{1}>
$$

where the matrix element of the t-matrix satisfies,

$$
\left\langle\varphi_{2}|T| \varphi_{1}\right\rangle=\left\langle\varphi_{2}|V| \psi_{1}^{(+)}\right\rangle=\left\langle\psi_{2}^{(-)}|V| \varphi_{1}\right\rangle
$$

Taking into account that the flux density of the incident beam of particles is $\left(\hbar k_{1} / m\right)$ the cross-section is found to be,

$$
(\mathrm{d} \sigma / \mathrm{d} \Omega)=\left(m / 2 \pi \hbar^{2}\right)^{2}\left(k_{2} / k_{1}\right)\left|\left\langle\varphi_{2}|T| \varphi_{1}\right\rangle\right|^{2}
$$

The distorted wave formulation of scattering theory hinges on finding a useful representation of $V$ as a sum of two potentials.

Let the two potentials be labelled by indices a and $b$. As before, states $\psi^{( \pm)}$are obtained from the Lippmann-Schwinger equation (B.2) only now $V=V_{\mathrm{a}}+V_{\mathrm{b}}$. One also defines new wave functions, $\chi^{( \pm)}$, for the potential $V_{\mathrm{a}}$, which then assumes the róle of the distorting potential,

$$
\chi_{2}^{(-)}=\varphi_{2}+\left(E-\mathrm{i} \eta-\mathscr{H}_{\mathrm{o}}\right)^{-1} V_{\mathrm{a}} \chi_{2}^{(-)}
$$

The key identity, obtained by straightforward manipulations, is, 


$$
\left\langle\varphi_{2}|V| \psi_{1}^{(+)}\right\rangle=\left\langle\chi_{2}^{(-)}\left|V_{\mathrm{a}}\right| \varphi_{1}\right\rangle+\left\langle\chi_{2}^{(-)}\left|V_{\mathrm{b}}\right| \psi_{1}^{(+)}\right\rangle
$$

To digest the physical significance of this identity the following observations might be helpful.

If $V_{\mathrm{b}}=0$ an inspection of (B.6) and (B.7) reveals that we recover the standard expression for the t-matrix associated with the potential $V_{\mathrm{a}}$, cf: (B.4). Looking next at the second term on the right-hand side of (B.7) we can make good use of the result,

$$
\psi^{(+)}=\varphi+(E+\mathrm{i} \eta-\mathscr{H})^{-1} V \varphi
$$

and the corresponding result for $\chi^{(+)}$, namely,

$$
\chi^{(+)}=\varphi+\left(E+\mathrm{i} \eta-\varkappa_{\mathrm{o}}-V_{\mathrm{a}}\right)^{-1} V_{\mathrm{a}} \varphi
$$

On subtracting these results and employing the operator identity,

$$
\left(E-H^{-1}\right)^{-1}\left(E-\mathscr{H}_{\mathrm{o}}-V_{\mathrm{a}}\right)^{-1}=\left(E-H_{f^{-1}} V_{\mathrm{b}}\left(E-\mathscr{H}_{\mathrm{o}}-V_{\mathrm{a}}\right)^{-1},\right.
$$

we arrive at the useful result,

$$
\psi^{(+)}=\chi^{(+)}+\left(E+\mathrm{i} \eta-H^{-1}\right)^{-1} V_{\mathrm{b}} \chi^{(+)} \equiv \chi^{(+)}+\left(E+\mathrm{i} \eta-H_{\mathrm{o}}-V_{\mathrm{a}}\right)^{-1} V_{\mathrm{b}} \psi^{(+)}
$$

From these results we see that $\psi^{(+)}$arises from the scattering of $\chi^{(+)}$by $V_{b}$, where $\chi^{(+)}$is the solution of the scattering problem defined by the potential $V_{\mathrm{a}}$. This observation engenders credence to the notion that $V_{\mathrm{a}}$ is our distorting potential.

At the first level of approximation in $V_{\mathrm{b}}$, we obtain from (B.10),

$$
\left\langle\chi_{2}^{(-)}\left|V_{\mathbf{b}}\right| \psi_{1}^{(+)}\right\rangle \sim\left\langle\chi_{2}^{(-)}\left|V_{\mathbf{b}}\right| \chi_{1}^{(+)}\right\rangle
$$


Inserting this in (B.7) leads to a result for the t-matrix which is frequently referred to as the distorted wave Born approximation. Results derived in the main text of the paper are based on the approximation given in (B.11), 


\section{Appendix C}

\section{Coherence Phenomena in Surface Scattering}

It seems appropriate in a discussion of optical properties of scattering by a surface to comment on pertinent features of coherence phenomena. For one thing, comments in the existing literature are more or less a farrago of sometimes misleading statements. Even so, the present discussion is very brief, for fear of inducing ennui, and it is certainly not put forward as an aperçu.

We have in mind experiments performed on the instrument CRISP situated at the ISIS facility: The incident neutrons emerge from a moderator that generates a pulse width, $\Delta t_{m}$, which has a maximum value of $150 \mu$ s achieved for a wavelength $\lambda=6.5 \AA$. Thereafter, the beam en route to the sample passes through a very broad-band chopper, and then impinges on the sample, located in the horizontal plane, through a collimator. The latter has a length of $2.5 \mathrm{~m}$, and a slit of variable dimension, $0.2-4.0 \mathrm{~mm}$, in height, and a fixed width $=40 \mathrm{~mm}$. Fixed detectors record the scattered signal in bins of width $20 \mu \mathrm{s}$, which is small compared to the pulse width at the moderator. It is also significant that, $\Delta t_{m}$ is very small compared to the openingtime of the broad-band chopper situated before the collimator.

Let us begin by estimating the coherence lengths associated with the incident beam. First, though, two general observations: coherence lengths in neutron beams incident on a sample are usually very small compared to those found in corresponding $\mathrm{x}$-ray instruments; and, an upper limit on the coherence length of a neutron beam is a few cm which is determined by the neutron half-life $\sim 10 \mathrm{~min}$.

Generally speaking, the degree of coherence between two points in the field of radiation can be influenced by two things: the average length of the wave packet of the radiation and the size of the source. The former is directly related to the spectral bandwidth of the source and the latter chiefly introduces diffraction effects which can degrade the visibility of interference effects, i.e. degrade the degree of coherence. 
Returning to CRISP, this may be regarded as a reasonably quasi-monochromatic instrument due to the accuracy of the time of flight determination. This means that the degree of lateral coherence is mainly limited by the divergence of the beam which determines the lateral spread in wave vectors. This in turn is half the inverse of the coherence length. The divergence perpendicular to the surface of the sample is typically $2 /\left(2.5 \cdot 10^{3}\right)$. For a wavelength of $6.5 \AA$ this gives an estimated perpendicular coherence length, $l_{\perp} \sim 10^{3} \AA$. (Here we are taking advantage of the fact that the incident beam is at a grazing angle $\theta \sim 1^{\circ}$.) $l_{\perp}$ is relevant when probing structures perpendicular to the sample surface without splitting the beam. Empirical evidence gathered over several years on CRISP is entirely consistent with this estimate.

When probing structures parallel to the sample surface, these are practically on the same line from the source due to the grazing incidence. In this case the degree of coherence is dominated by temporal effects and is therefore related to the effective bandwidth set by the time of flight accuracy. The same applies for multiple reflection effects since points along the reflected beams essentially lie on the same line and have been separated only because the original beam has been split. We have for the parallel coherence length,

$$
l_{/ /} \sim \frac{\lambda^{2}}{\Delta \lambda}=\frac{\lambda}{\frac{\Delta t}{t}+\frac{\Delta s}{s}},
$$

where $\lambda$ is the wavelength, and $\Delta t / t$ and $\Delta s / s$ are the relative uncertainties in the time of flight and the path-length, over which the time of flight is measured, respectively. The latter uncertainty has negligible influence, only about a part in a million, due to the rather long path-length $(>10 \mathrm{~m})$, and the coherence length is therefore limited by $\Delta t / t$. $\Delta t$ saturates at a value of $120 \mu \mathrm{s}$ for the longer wavelengths, and for $\lambda=6.5 \AA$ the time of flight is about $20 \cdot 10^{3} \mu$ s. This yields an estimate $l_{l /} \sim 10^{3} \AA$, which is in accord with empirical evidence from CRISP. In this context it is worth mentioning that, on the basis of experimental results, the coherence time, which is directly proportional to $l_{l l}$ and hence inversely proportional to $\Delta t$, has an upper limit of about $10^{-3} \mu \mathrm{s}$. The 
influence of the various time windows (moderator, bin width, chopper) on the average length of the wave packet is therefore negligible. 


\section{Table 1}

Selected examples of Bessel transforms of isotropic autocorrelation functions.

\begin{tabular}{|ll|l|}
\hline & $C(\rho)$ & $\int_{0}^{\infty} \mathrm{d} \rho^{2} J_{0}(\kappa \rho) C(\rho)$ \\
\hline 1. & $\exp \left(-\rho^{2} / \xi^{2}\right)$ & $\xi^{2} \exp \left(-\kappa^{2} \xi^{2} / 4\right)$ \\
2. & $\alpha^{3} /\left(\rho^{2}+\alpha^{2}\right)^{3 / 2}$ & $2 \alpha^{2} \exp (-\kappa \alpha)$ \\
3. & $\exp (-\rho / \xi)$ & $2 \xi^{2} /\left\{(\kappa \xi)^{2}+1\right\}^{3 / 2}$ \\
4. & $J_{0}(2 \rho / \lambda) \exp \left(-\rho^{2} / \xi^{2}\right)$ \\
5. & $j_{0}(\rho / \lambda)$ & $\xi^{2} I_{0}\left(\xi^{2} \kappa / \lambda\right) \exp \left\{-\xi^{2}\left(\kappa^{2}+(2 / \lambda)^{2}\right) / 4\right\}$ \\
& $\left\{\begin{array}{l}2 \lambda^{2} /\left\{1-(\kappa \lambda)^{2}\right\}^{1 / 2} ; \kappa \lambda \leq 1 \\
0 \quad ; \kappa \lambda>1\end{array}\right.$ \\
\hline
\end{tabular}




\section{Figure Captions}

(1.) A plane wave is incident at a grazing angle $\theta$ to a surface defined by the $x-y$ plane. The plane of incidence coincides with the $x$-axis. The region $z>0$ is a vacuum half-space in which the refractive index is unity, and the half-space $z<0$ is occupied by a refractive medium. The wave vector of the incident wave is expressed in the form $\mathbf{k}=\left(k_{x}, k_{y},-k_{z}\right)$, cf. Appendix A.

(2.) A rough surface is defined by $\zeta(x, y)$ which lies in the half-space $z<0$. Hence, the perturbing potential in our application of the distorted wave formulation of scattering theory is $-V_{0}$ for $-\zeta \leq z \leq 0$ and zero elsewhere, where $V_{0}$ is the material optical potential equ (2.2).

(3.) a) Specular reflectivity, for neutrons, of a piecewise rectangular and a sinusoidal grating as obtained from the distorted wave Born approximation using the Fermi psuedo-potential plotted against the perpendicular wave vector transfer. In comparison the reflectivity for an optical flat is also shown. b) Same as a) showing the effect of increasing the maximum height variation and decreasing the scattering length density which in this case is that of nickel.

(4.) Specular reflectivity for two piecewise rectangular gratings with different widths of the ridge in comparison to the spatial wavelength of the grating plotted against the perpendicular wave vector transfer. The maximum deviation from the optical flat reflectivity occurs when the ridge is as wide as the trough.

(5.) Specular reflectivity of a piecewise rectangular grating and that of a film on top of a bulk as obtained from Airy's formula including multiple reflections plotted against the perpendicular wave vector transfer. The film has half the scattering length density of the bulk. 
(6.) a) First order diffuse reflectivities for a piecewise rectangular and a sinusoidal grating for an incident grazing angle of $1.5^{\circ}$ plotted against twice the $z$-component of the incident wave vector to facilitate comparison with specular reflectivity graphs. The right ordinate axis indicates the magnitude of the scattering angle measured from the surface. b) Same as a) but the scattering angle is larger than the incident angle as indicated by the negative order $n$.

(7.) Second order diffuse reflectivities for a sinusoidal grating for an incident grazing angle of $1.5^{\circ}$ plotted against twice the $z$-component of the incident wave vector. The right ordinate axis shows the scattering angle as measured from the surface.

(8.) Third order diffuse reflectivities for a piecewise rectangular and a sinusoidal grating for an incident grazing angle of $1.5^{\circ}$, cf. figs. ( $\left.6 \mathrm{a}, \mathrm{b}\right)$.

(9.) Specular reflectivity for a surface with a near Gaussian height distribution as approximated by the distorted wave Born approximation eq. (5.5). The dashed lines are the upper and lower envelopes given by $R\left\{1-\left(k_{z} / k_{z}^{t}\right)\left(1 \pm \exp \left\{-\frac{1}{2}(q, \sigma)^{2}\right\}\right)\right\}^{2}$.

(10.) Specular reflectivity for a Gaussian surface with a root-mean-square deviation, $\sigma$, of $10 \AA$ as given by the distorted wave Born approximation and the Born approximation.

(11.) Diffuse reflectivity for an isotropic surface with a Gaussian height distribution, $\sigma=10 \AA$, and a Gaussian autocorrelation function, labelled (1) in Table 1, in the region $\left(\sigma q_{t}\right) \leq 0.3$ plotted against the scattering angle $\theta_{2}$. The curves correspond to different values of the correlation length $\xi$.

(12.) Diffuse reflectivity for an isotropic surface with Gaussian height characteristics, $\sigma=10 \AA$, and a mixed autocorrelation function, labelled (4) in Table 1, plotted against the scattering angle $\theta_{2}$. The plotted range corresponds to $\left(\sigma q_{t}\right) \leq 0.3$. 
(13.) The various Fresnel wave functions used in the distorted wave Born approximation represented by arrows parallel to the propagation vectors of the constituents, cf. equs. (A.2), (A.7) and (A.9). $\chi_{1}^{(+)}$and $\chi_{2}^{(-)}$are relevant quantities in the derivation of specular and diffuse reflectivity whereas $\chi_{2 T}^{(-)}$rather than $\chi_{2}^{(-)}$is used to estimate specular and diffuse transmissivity. 


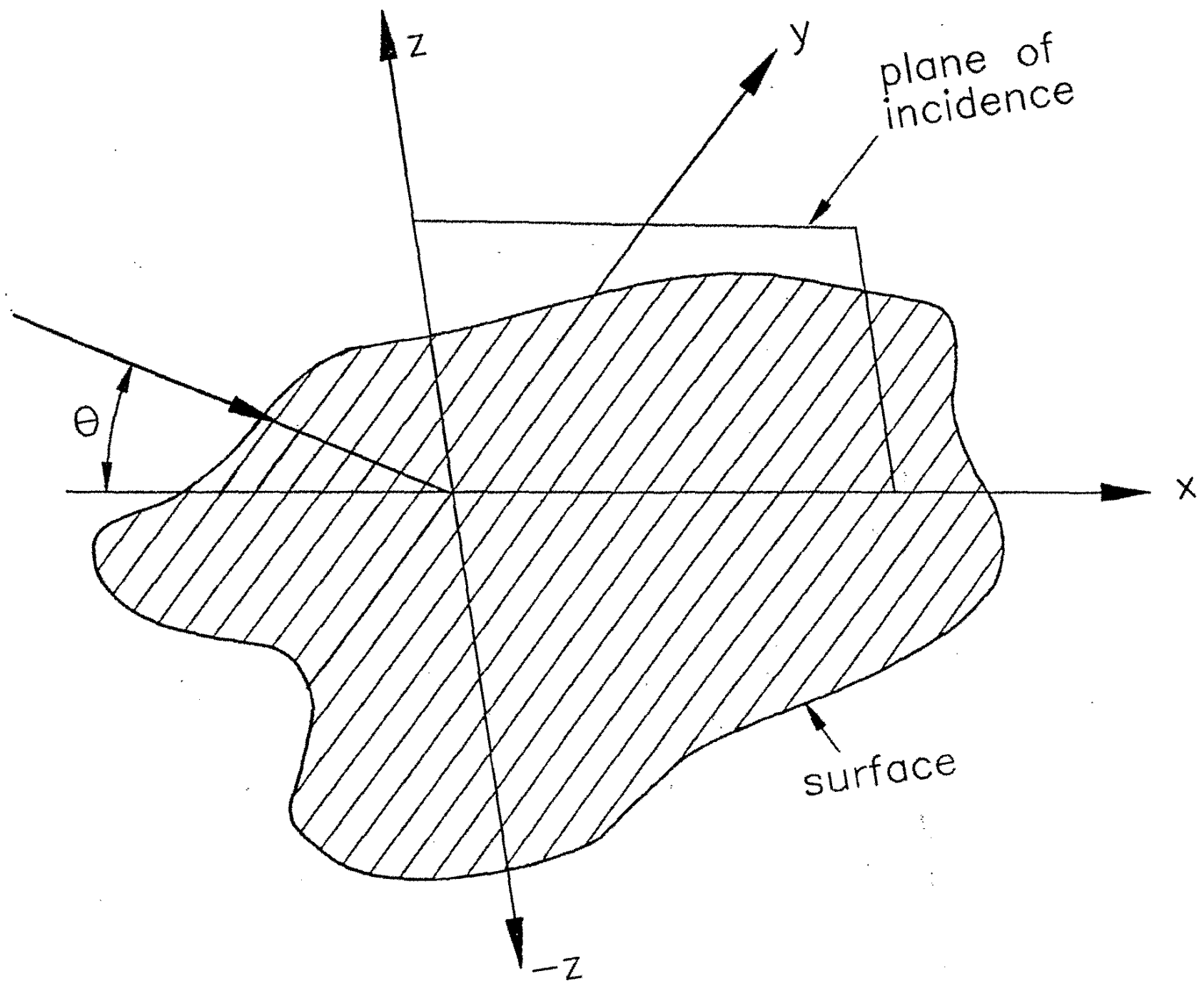

fig. 1 


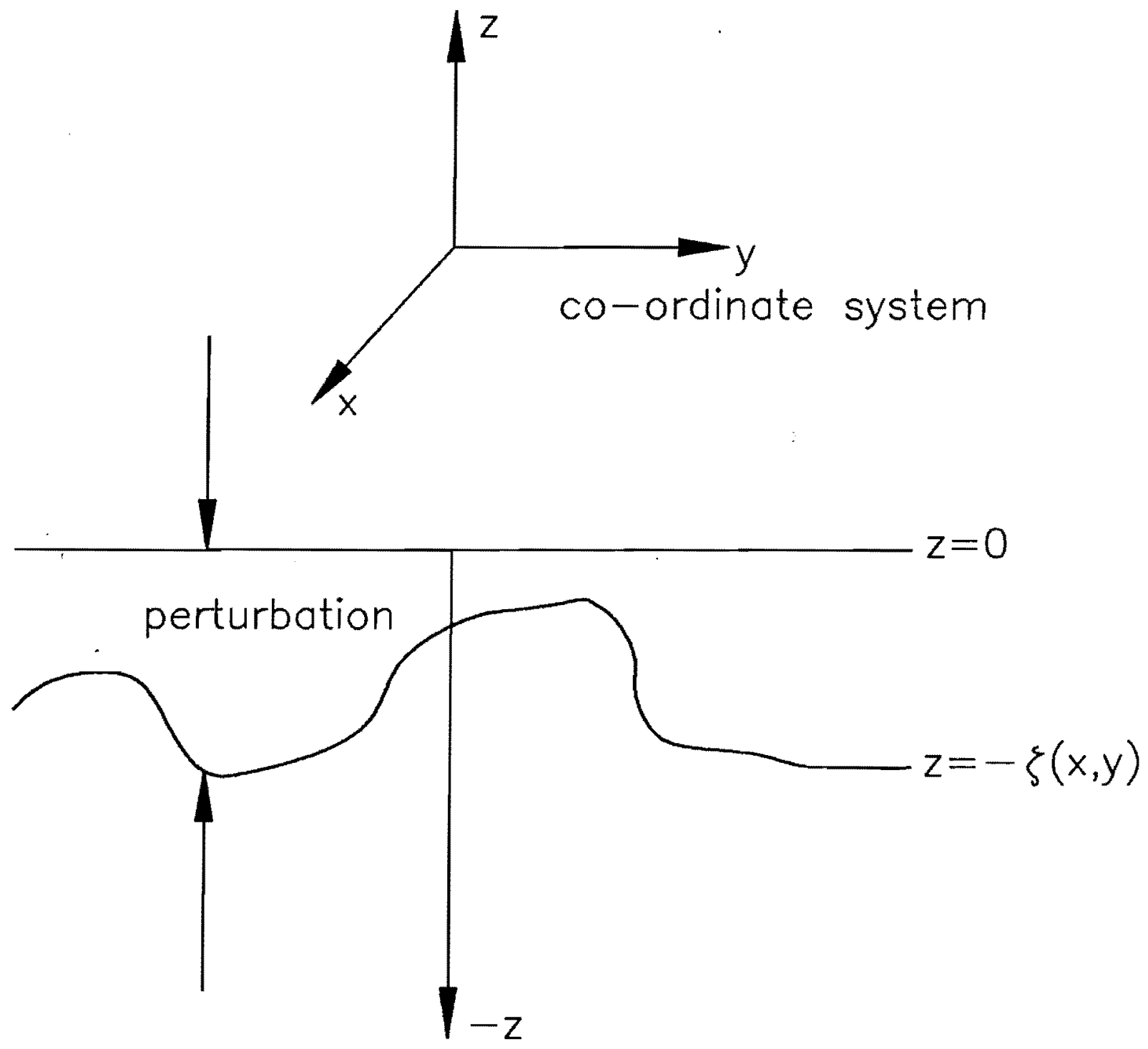

fig 2 
$h=5 A, N b=0.005 A^{-2}, q_{c}=0.501 A^{-1}$

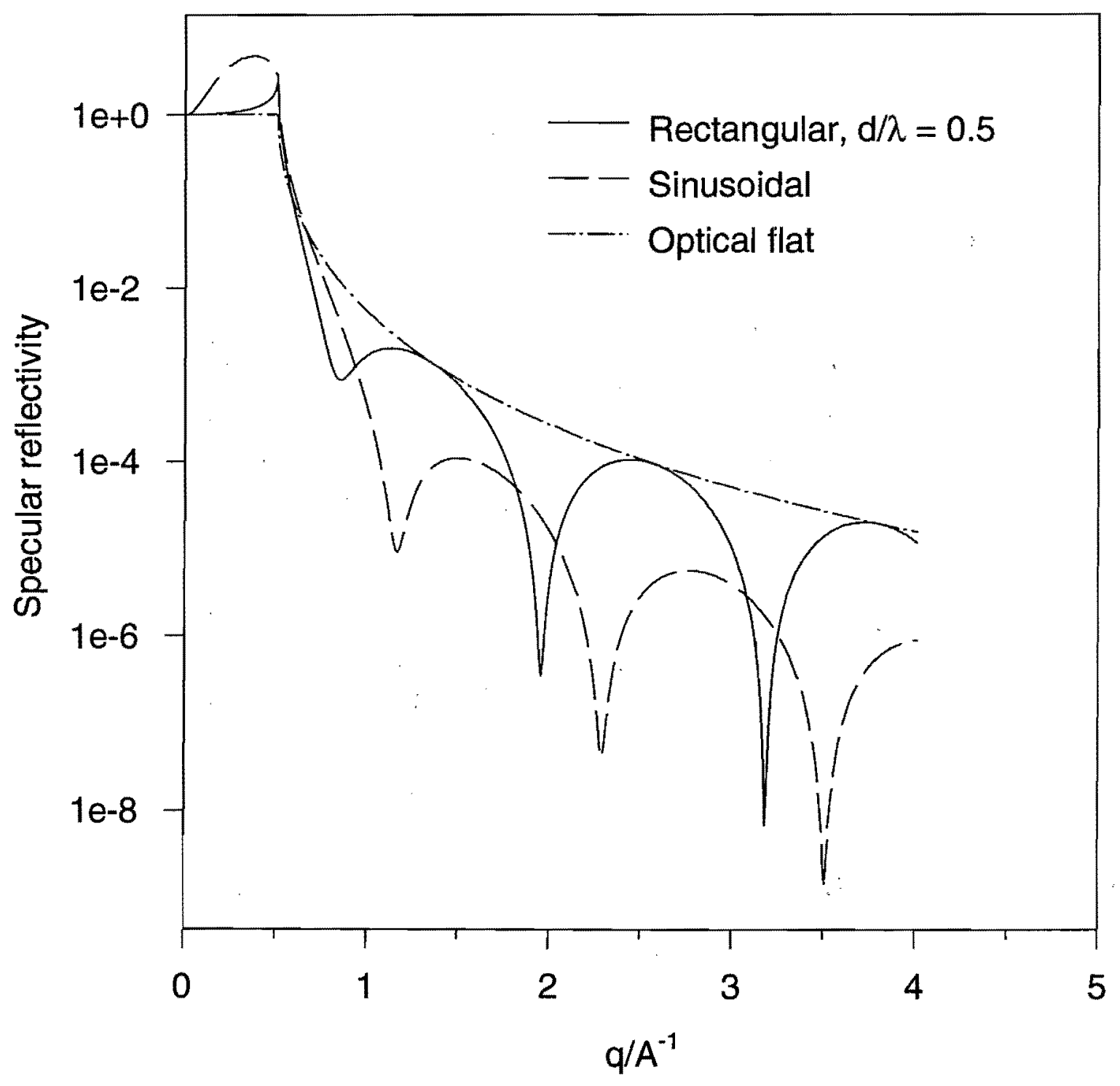

fig $3 a$ 


$$
h=500 A, N b=10^{-5} A^{-2}, q_{c}=2.2410^{-2} A^{-1}
$$

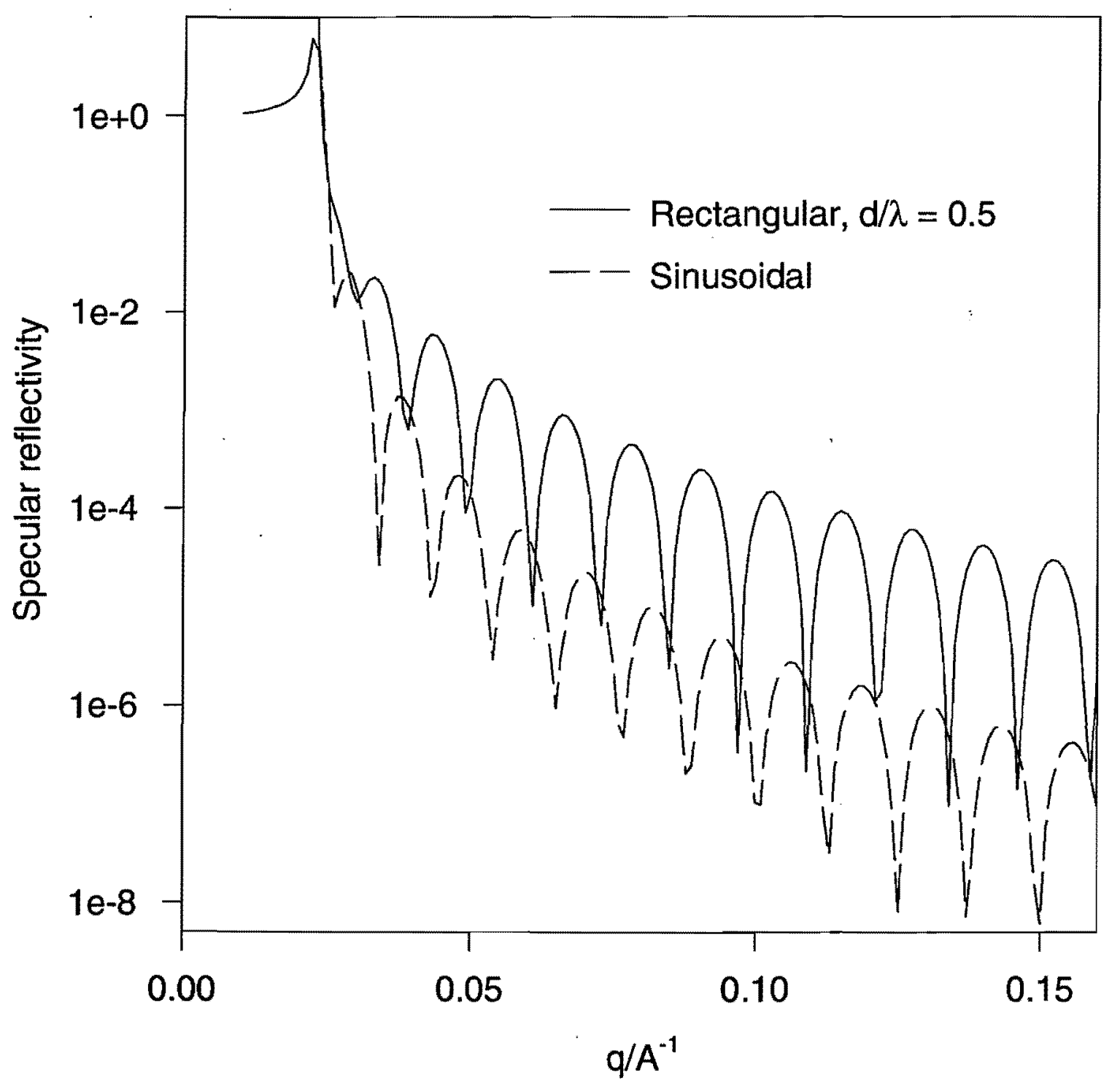

fig $3 b$ 
Rectangular: $h=5 \mathrm{~A}, \mathrm{Nb}=0.005 \mathrm{~A}^{-2}, q_{c}=0.501 \mathrm{~A}^{-1}$

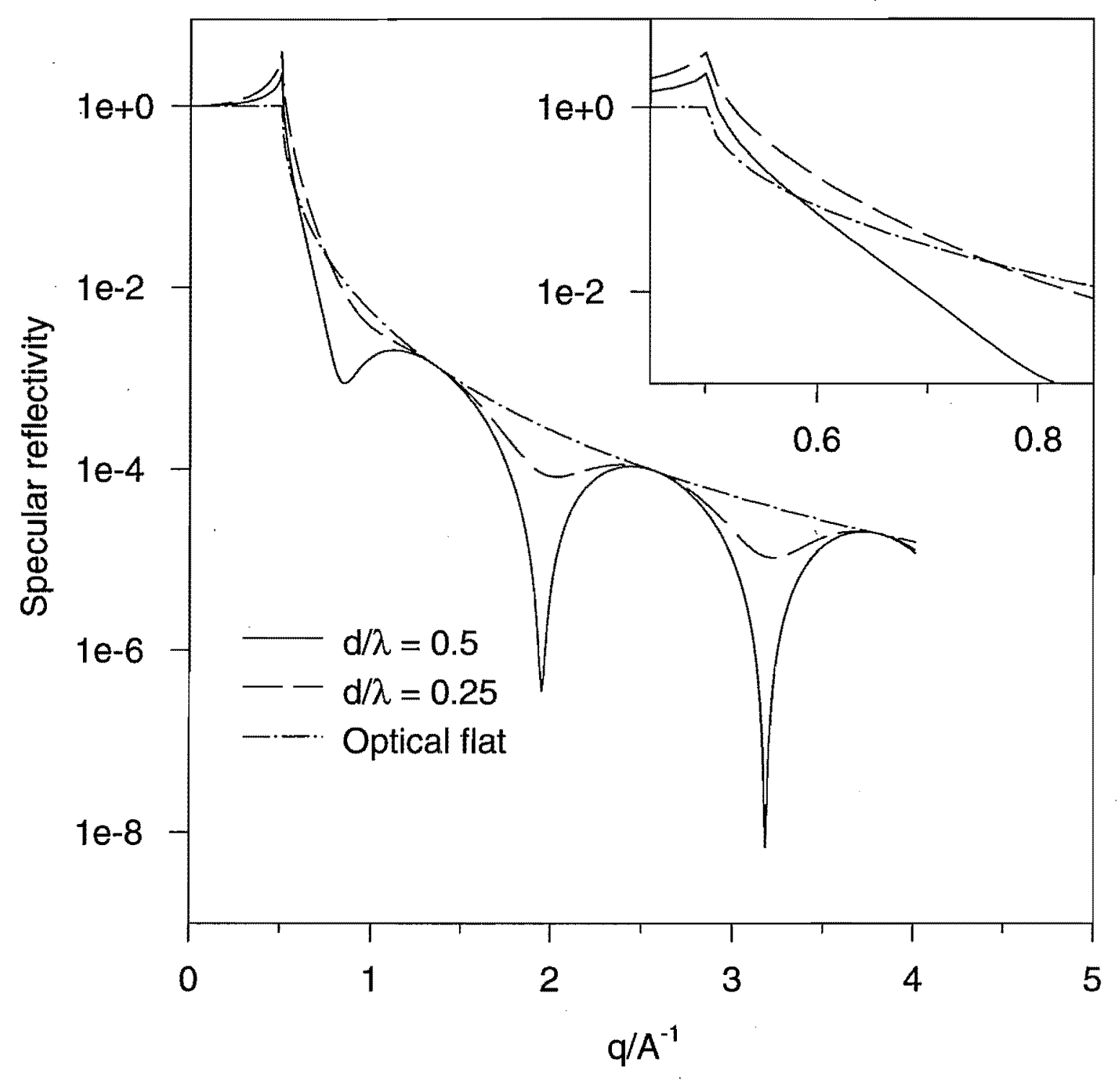

fig. 4 


$$
h=5 A, N b=0.005 A^{-2}, q_{c}=0.501 A^{-1}
$$

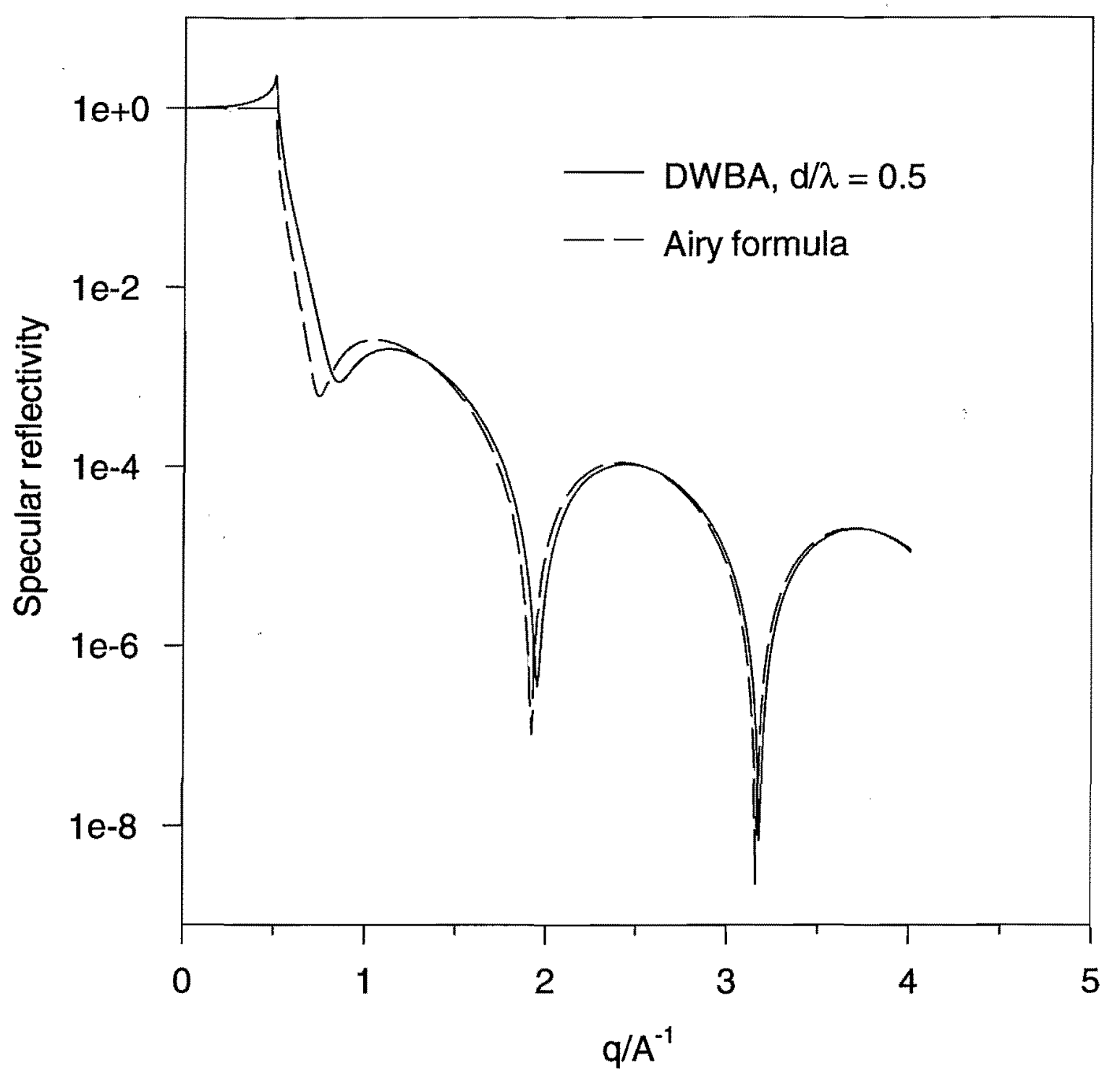

fig 5 


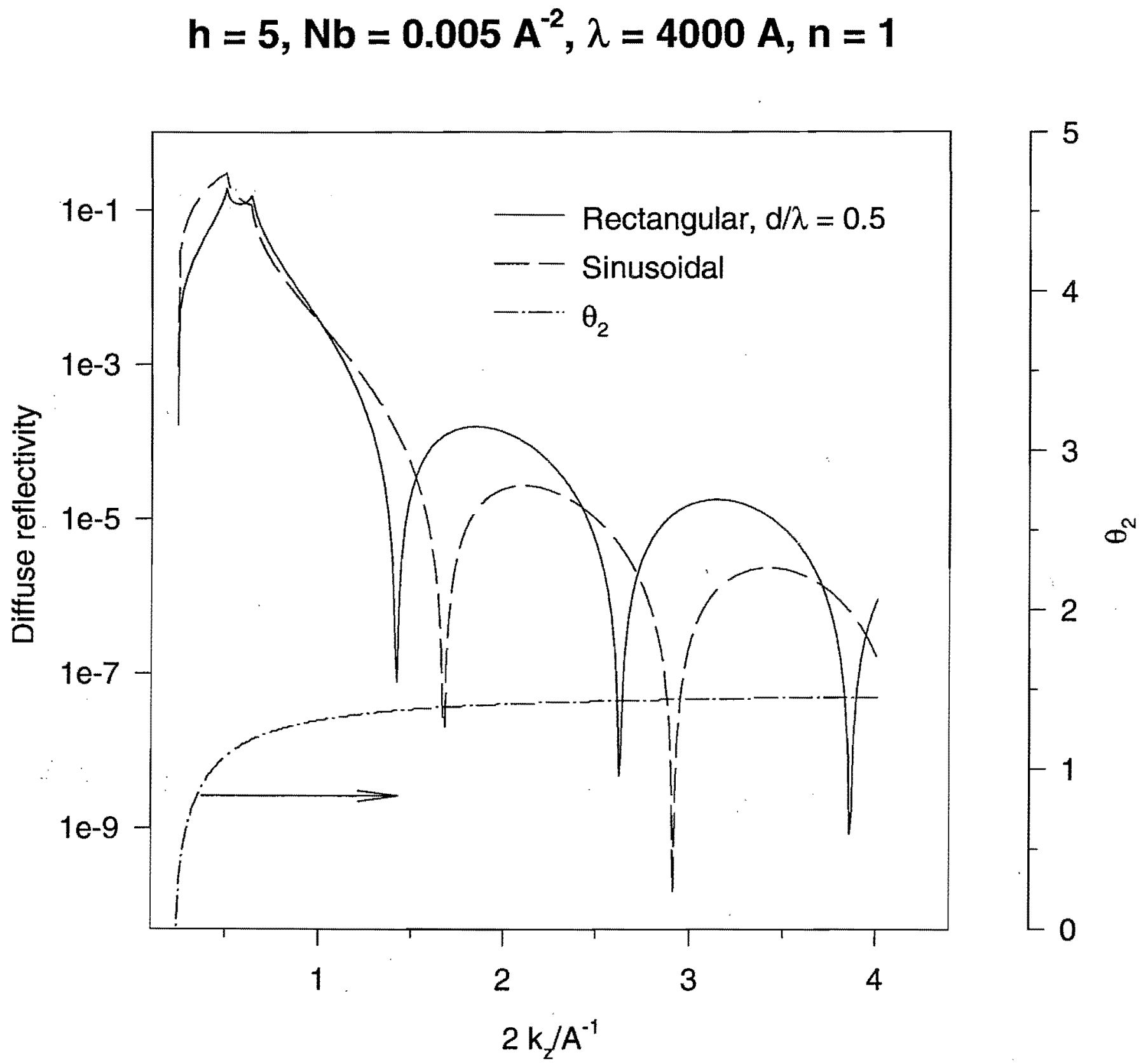

flg $6 a$ 


$$
h=5 A, N b=0.005 A^{-2}, \lambda=4000 A, n=-1
$$

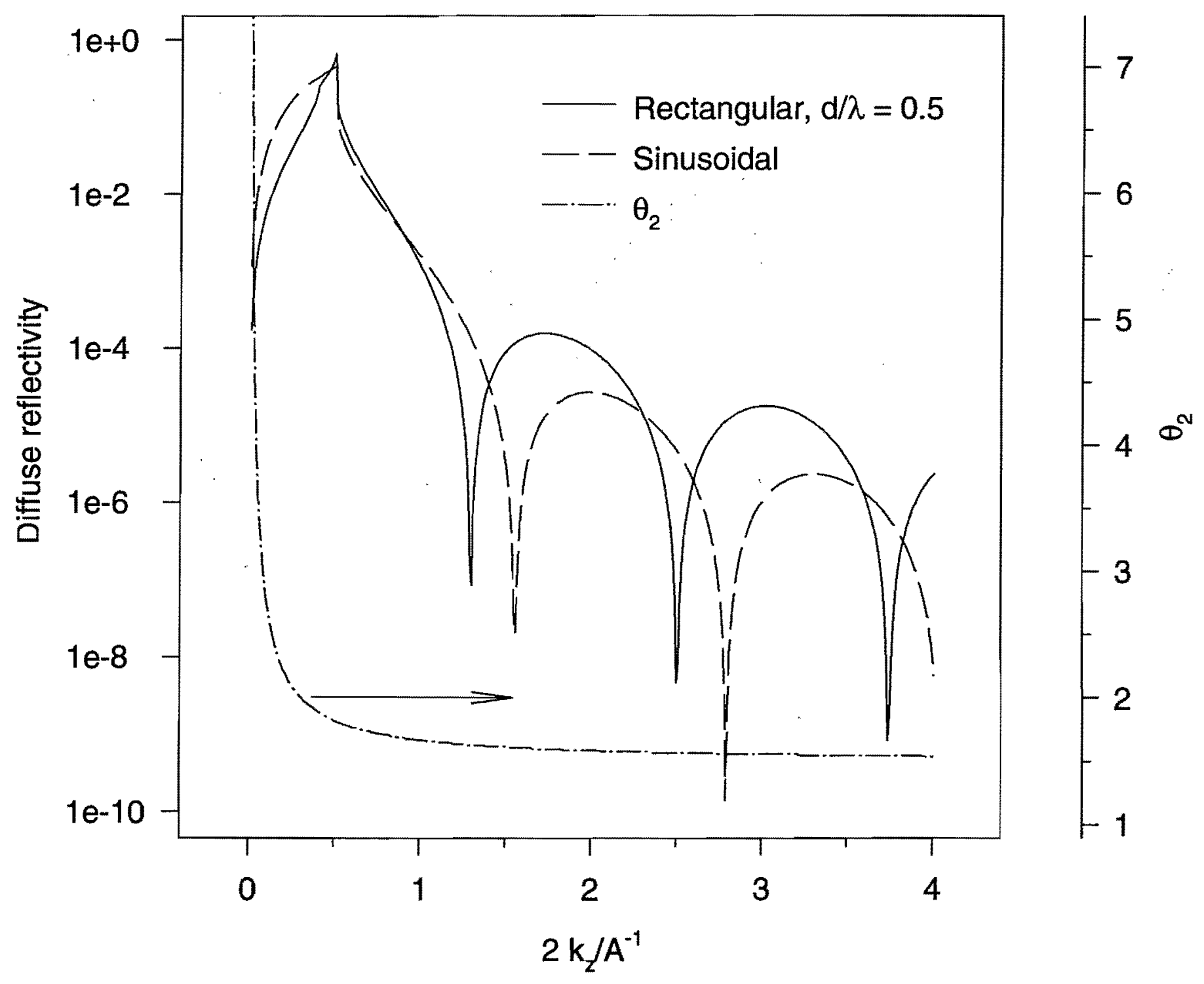

$\operatorname{tig} 66$ 


\section{Sinusoidal}

$h=5 \mathrm{~A}, \mathrm{Nb}=0.005 \mathrm{~A}^{-2}, \lambda=4000 \mathrm{~A}, \mathrm{n}=2,-2$

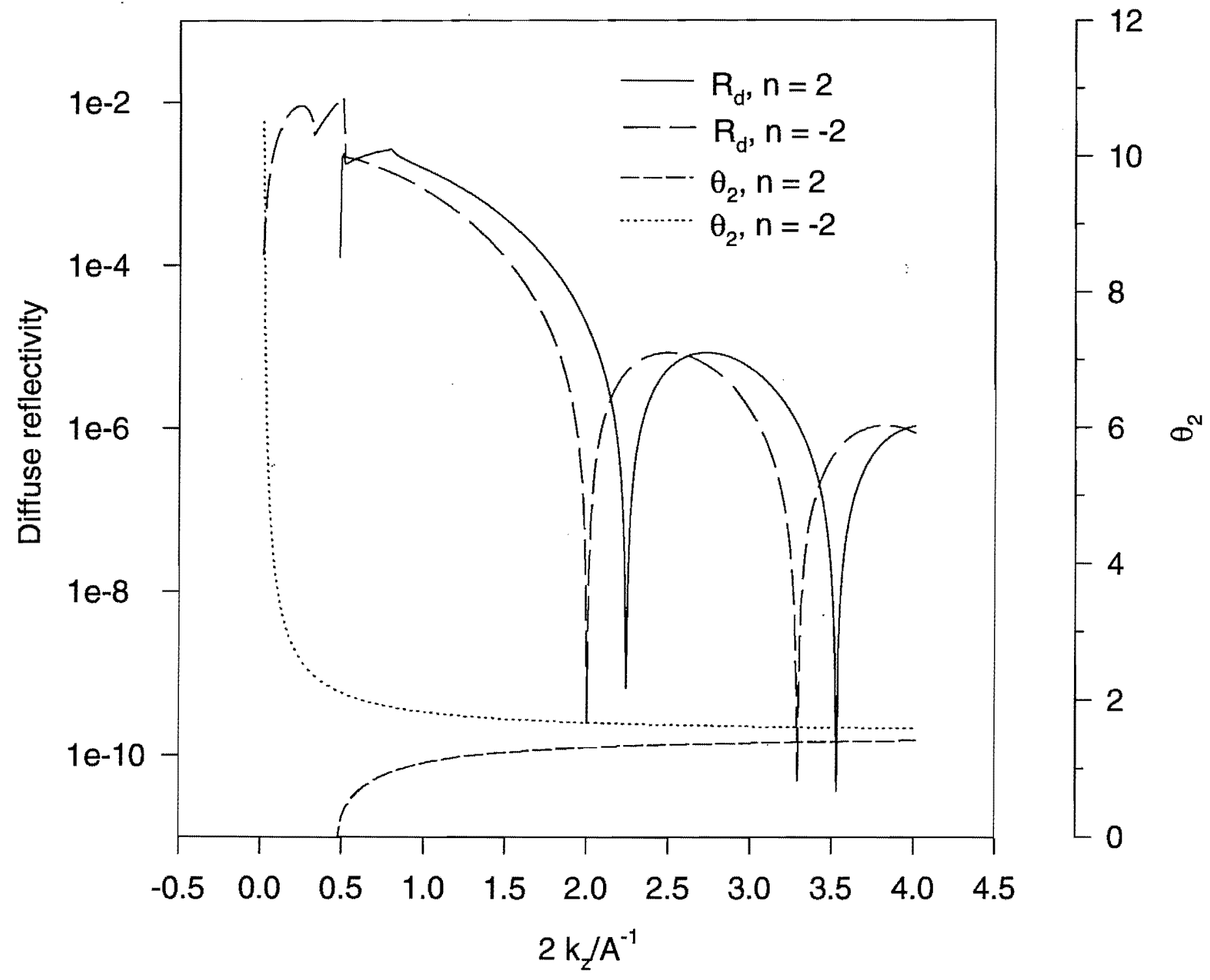

fig 7 


$$
h=5 A, N b=0.005 A^{-2}, \lambda=4000 A, n=3
$$

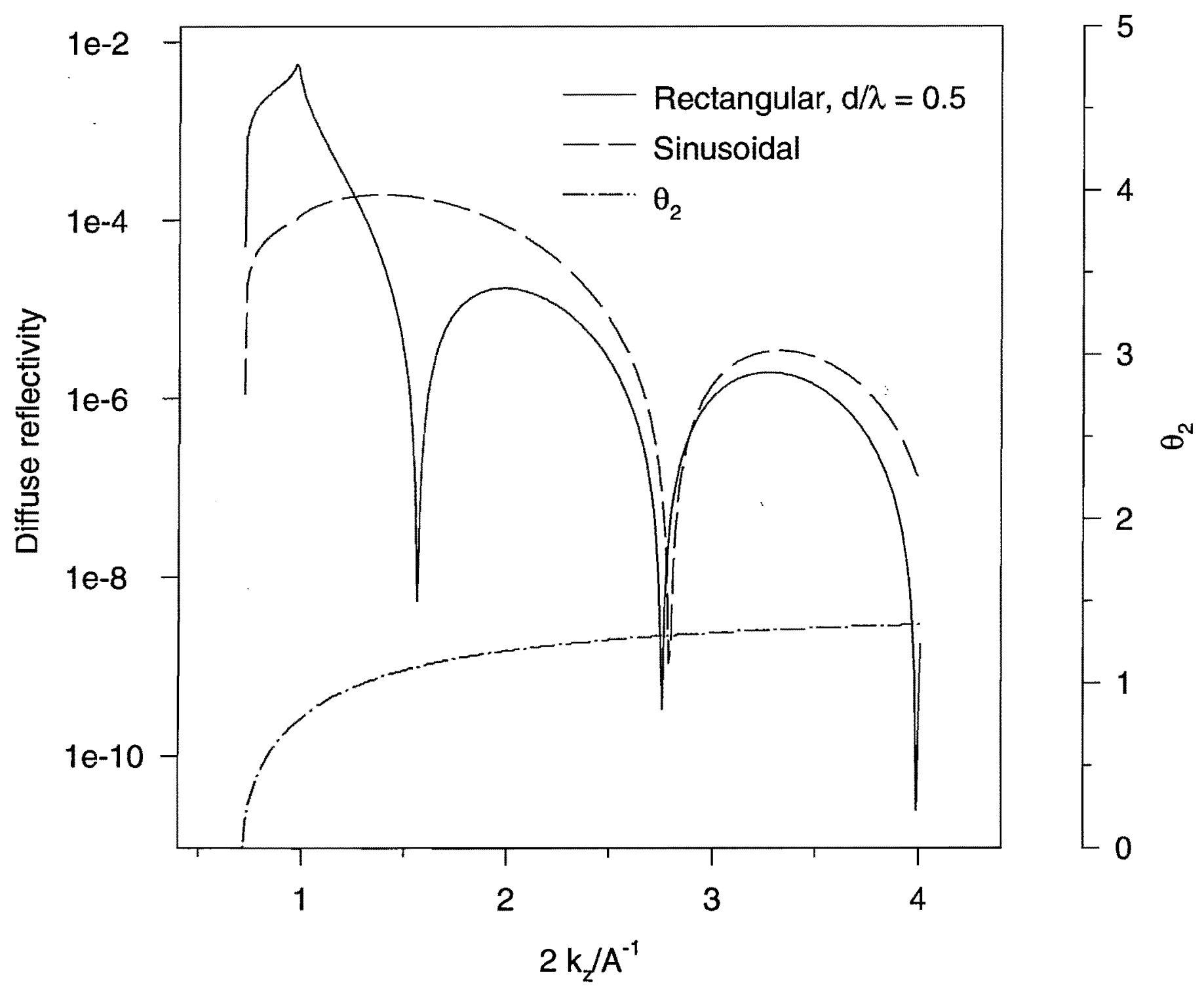

fig $8 a$ 
$h=5 A, N b=0.005 A^{-2}, \lambda=4000 A, n=-3$

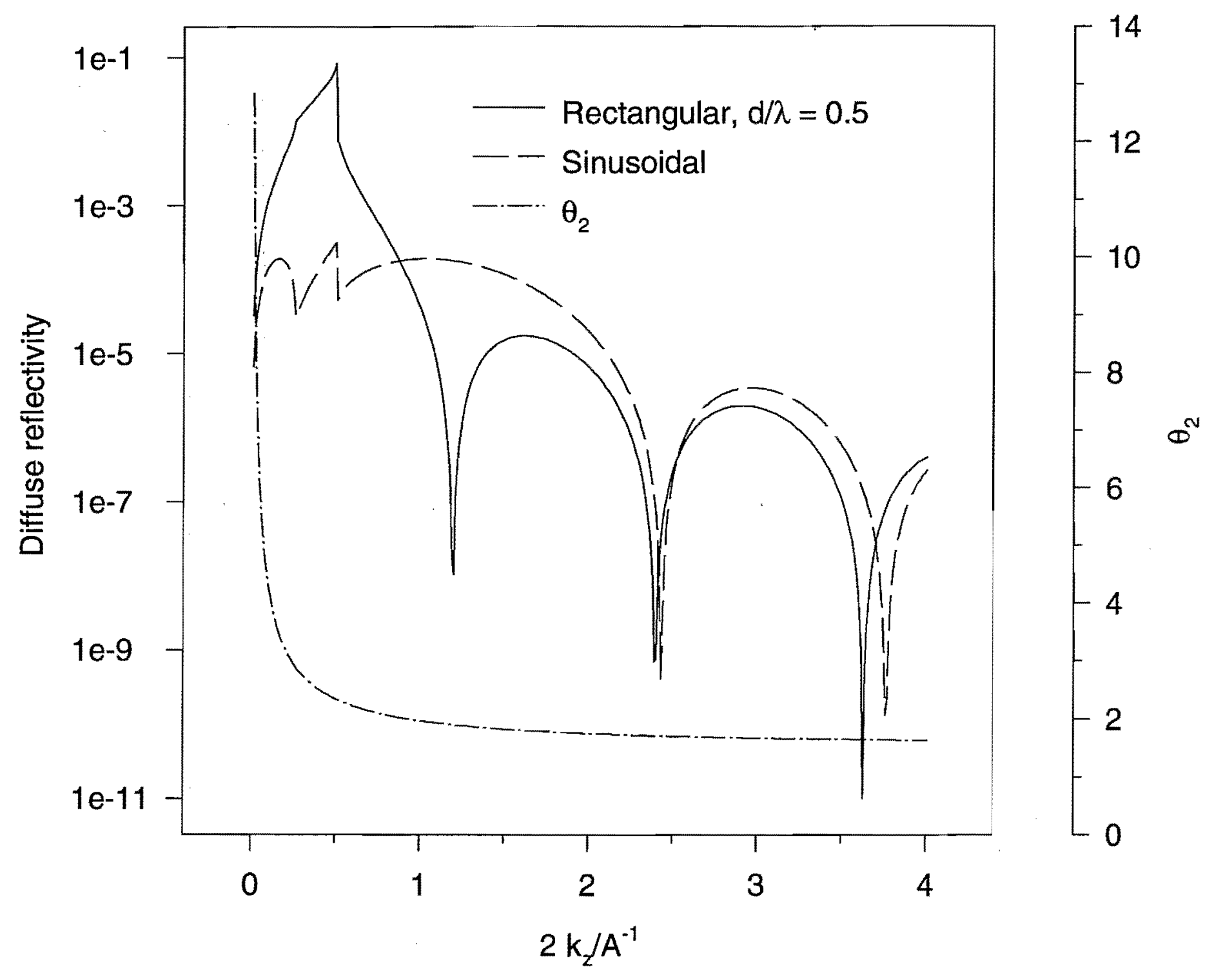

fig $8 b$ 
Gaussian: $<\zeta>=200 \mathrm{~A}, \sigma=10 \mathrm{~A}, \mathrm{Nb}=10^{-5} \mathrm{~A}^{-2}$

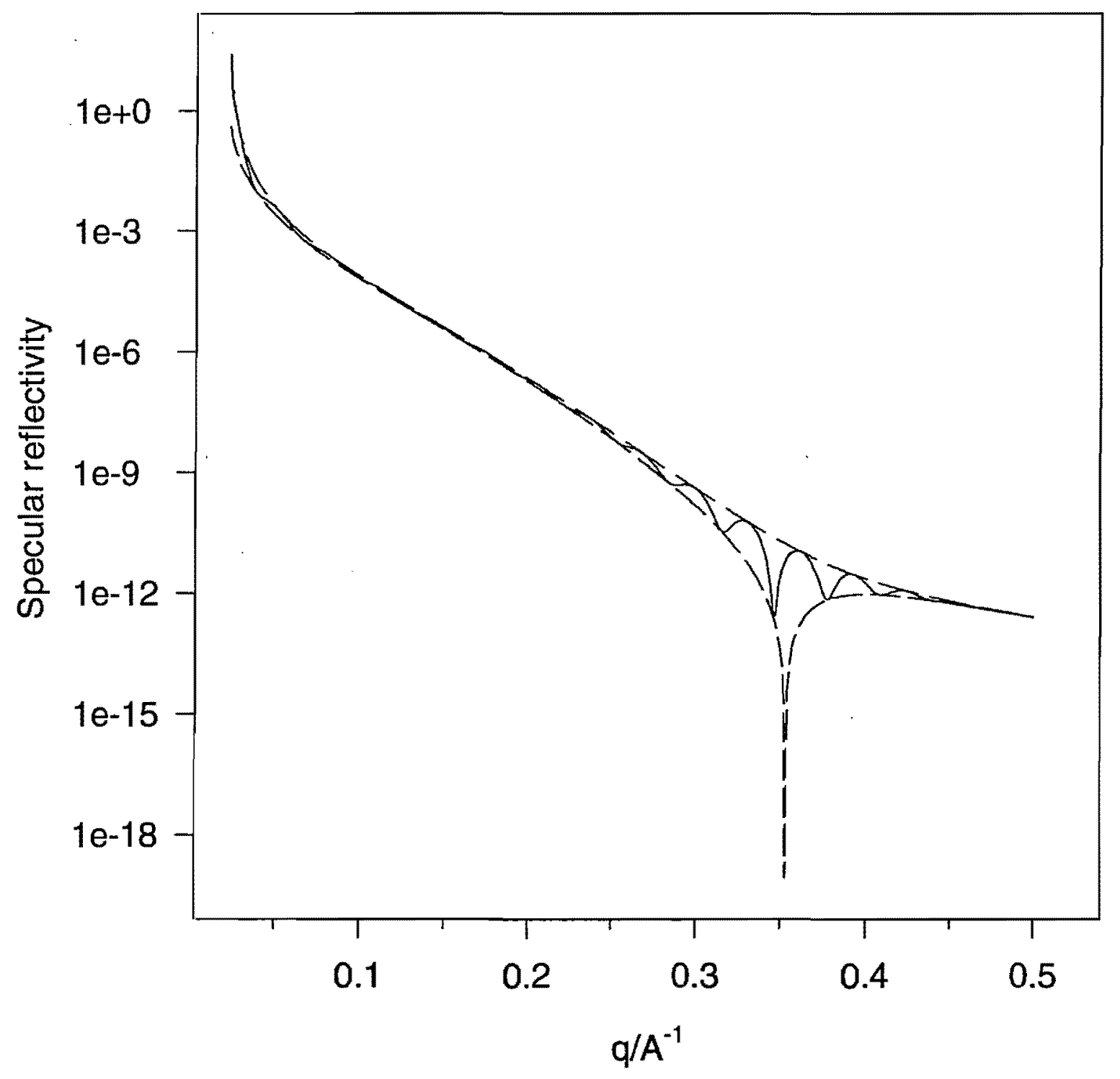

fig 9 
Gaussian: $\sigma=10 \mathrm{~A}, \mathrm{Nb}=10^{-5} \mathrm{~A}^{-2}$

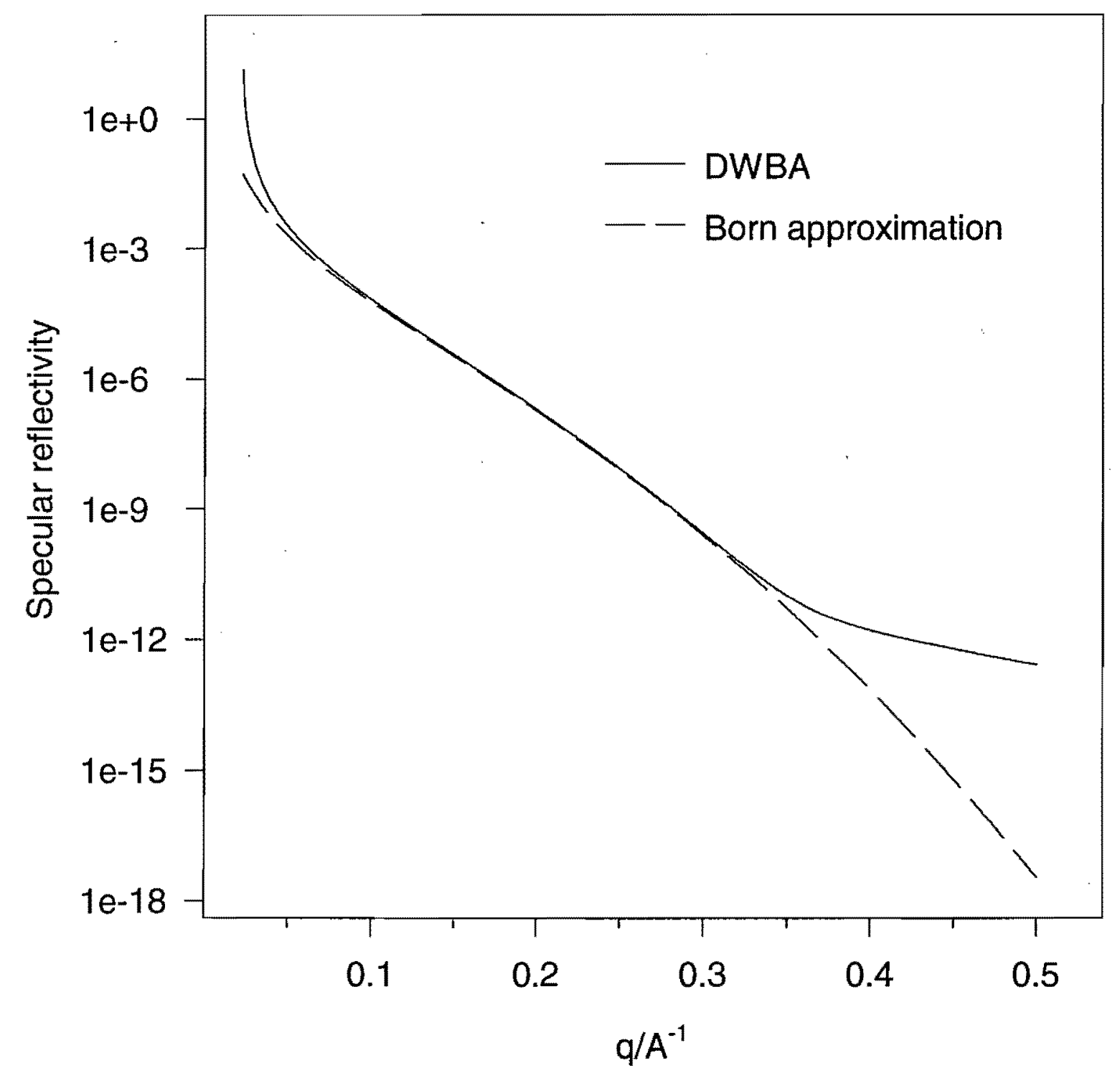

$f \lg 10$ 


\section{$C(\rho)=\exp \left(-\rho^{2} / \xi^{2}\right): \sigma=10 A, N b=10^{-6} A^{-2}, q=0.01 A^{-1}$}

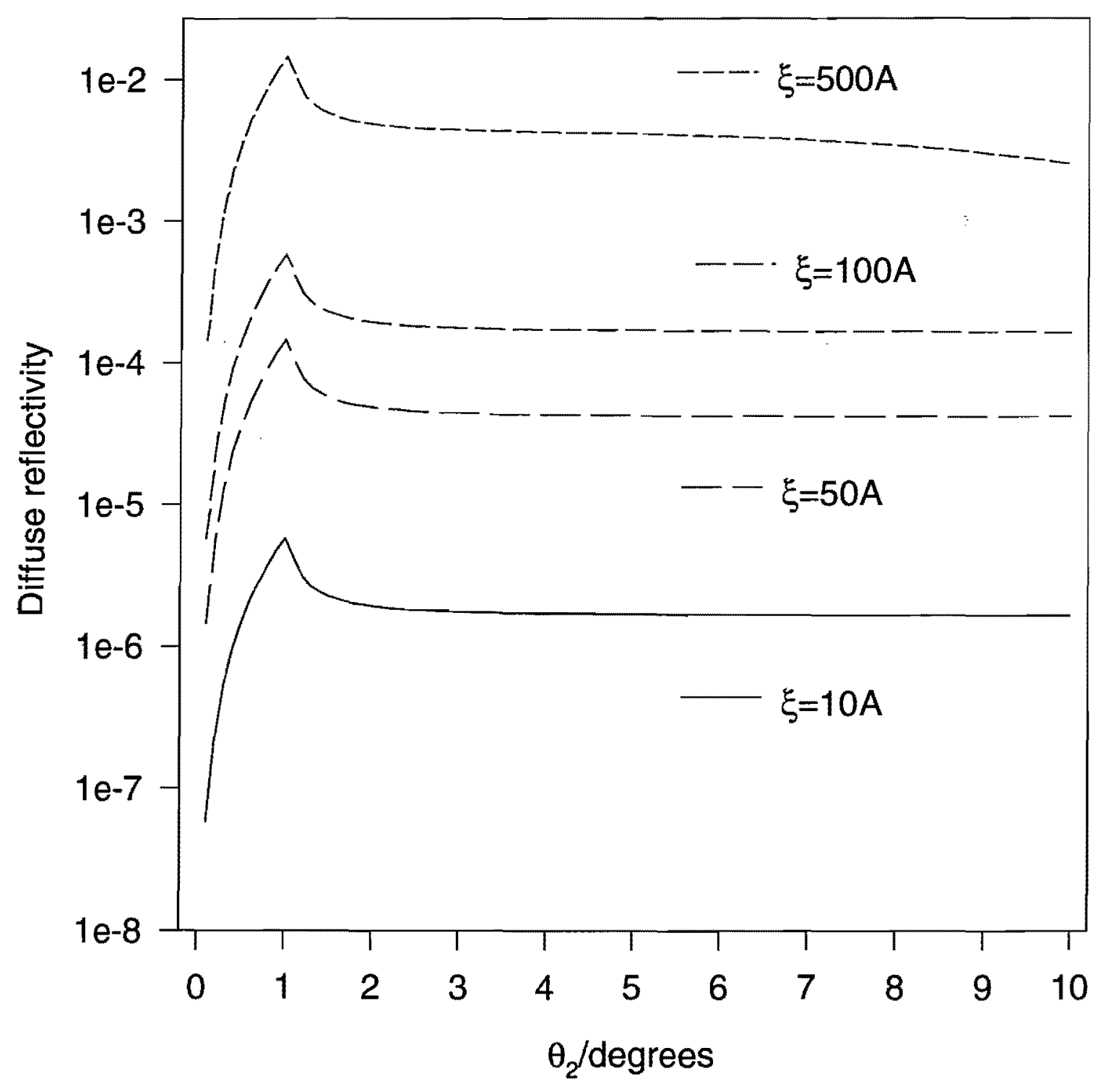

fig. II 
$C(\rho)=J_{0}(2 \rho / \lambda) \exp \left(-\rho^{2} / \xi^{2}\right): \sigma=10 A, N b=10^{-6} A^{-2}, q=0.01 A^{-1}$

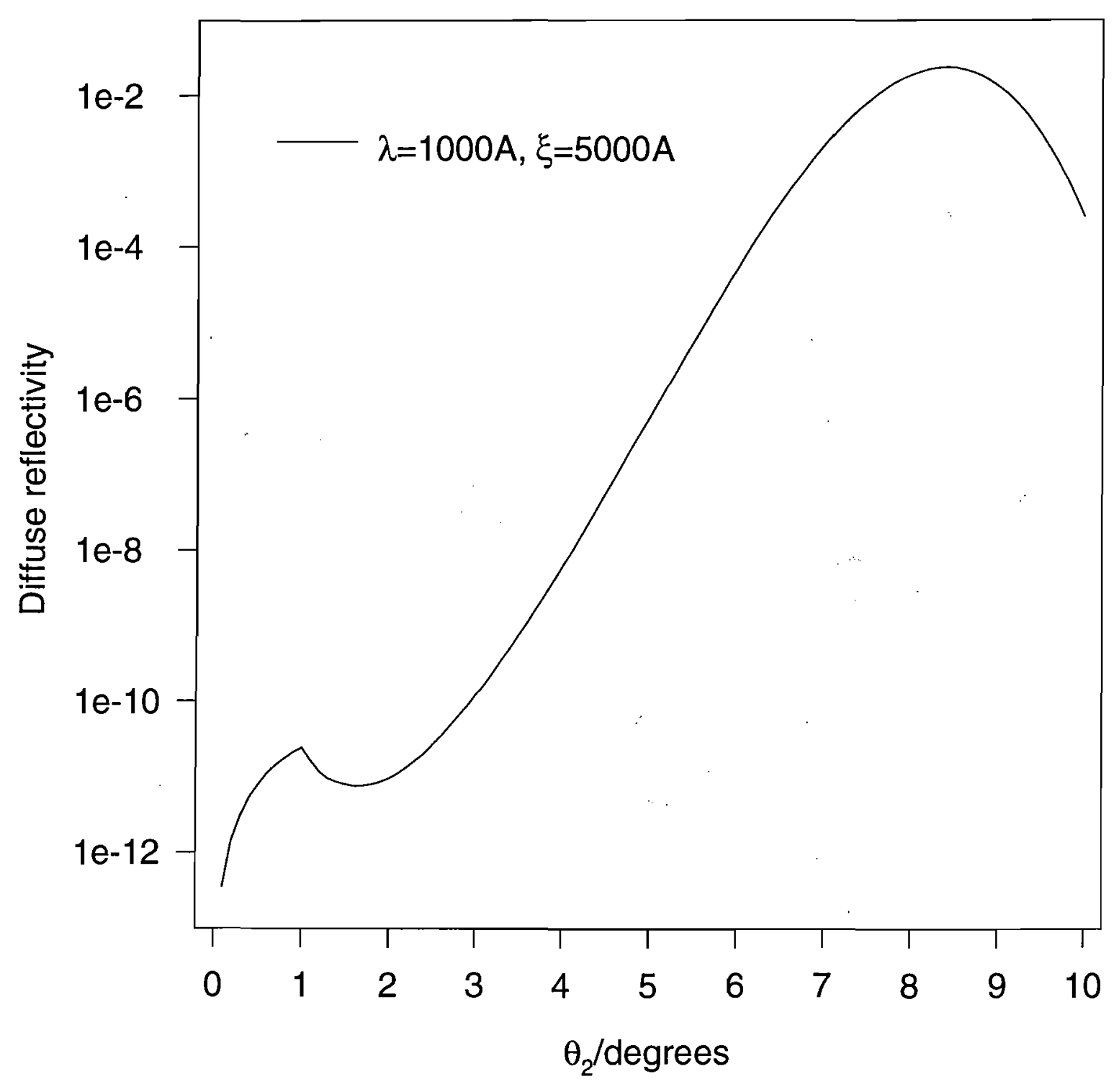

fig. 12 


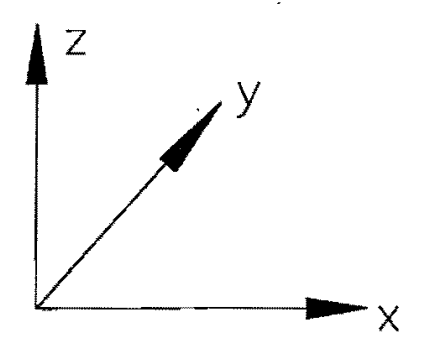

co-ordinate system

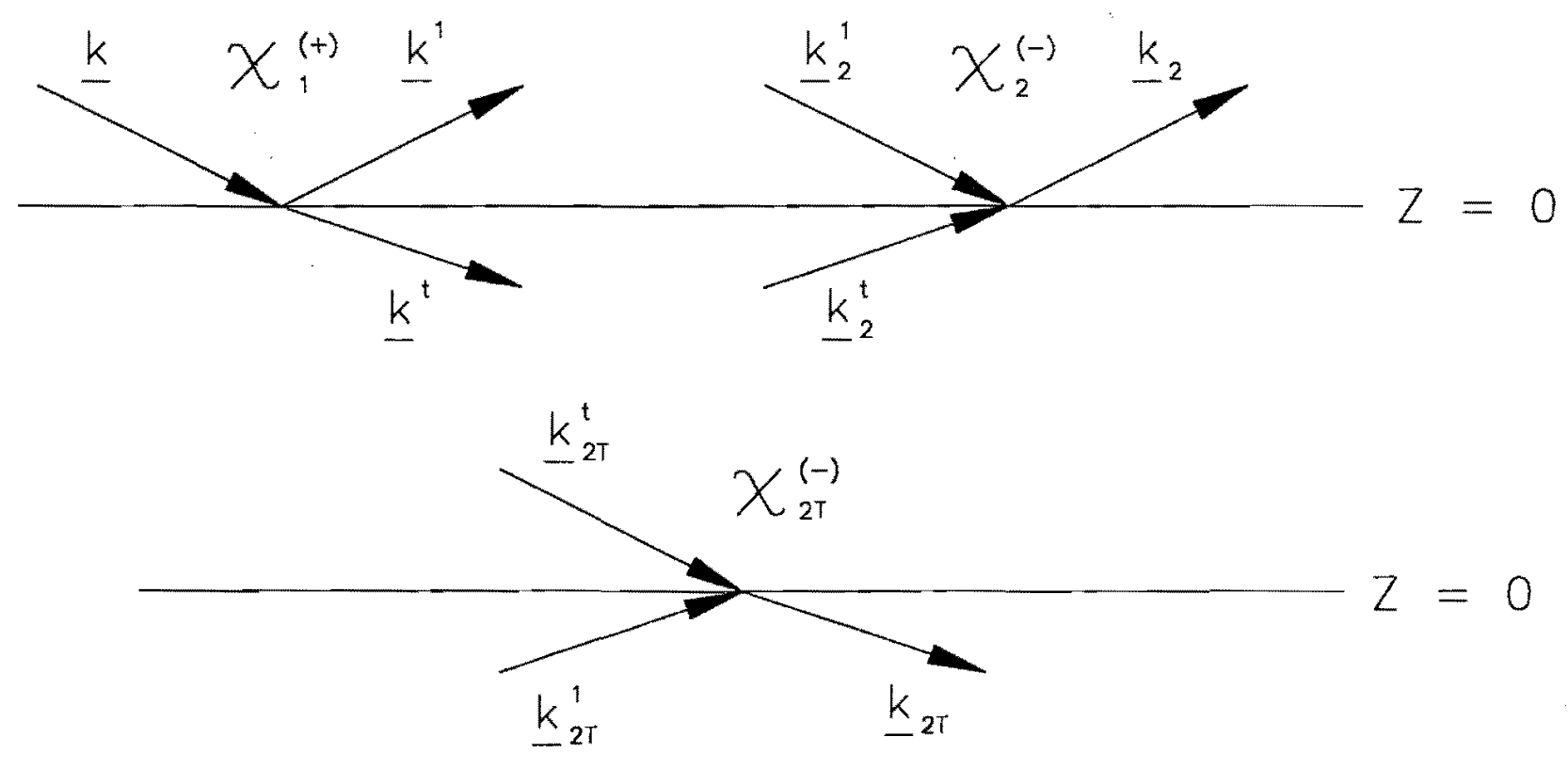

fig. 13 